Provided for non-commercial research and education use. Not for reproduction, distribution or commercial use.

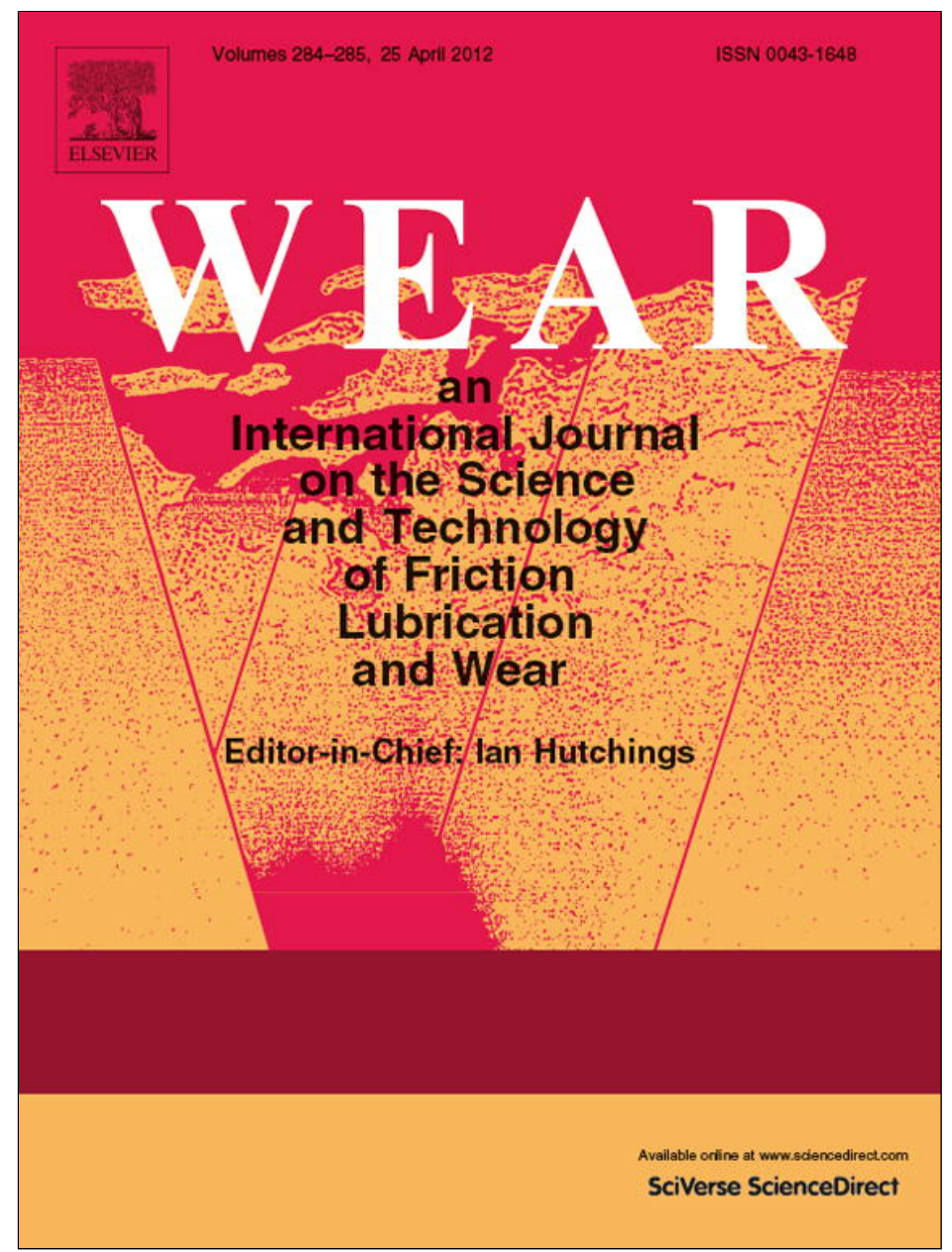

This article appeared in a journal published by Elsevier. The attached copy is furnished to the author for internal non-commercial research and education use, including for instruction at the authors institution and sharing with colleagues.

Other uses, including reproduction and distribution, or selling or licensing copies, or posting to personal, institutional or third party websites are prohibited.

In most cases authors are permitted to post their version of the article (e.g. in Word or Tex form) to their personal website or institutional repository. Authors requiring further information regarding Elsevier's archiving and manuscript policies are encouraged to visit:

http://www.elsevier.com/copyright 


\title{
Development of a wear model for the prediction of wheel and rail profile evolution in railway systems
}

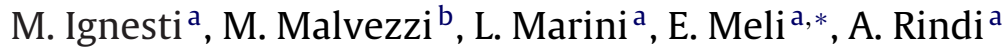 \\ a Department of Energy Engineering, University of Florence, Via S. Marta n. 3, 50139 Firenze, Italy \\ ${ }^{\mathrm{b}}$ Department of Information Engineering, University of Siena, Via Roma n. 56, 53100 Siena, Italy
}

\section{A R T I C L E I N F O}

Article history:

Received 25 May 2011

Received in revised form

22 December 2011

Accepted 3 January 2012

Available online 24 January 2012

\section{Keywords:}

Multibody modeling

Wheel-rail contact

Wheel-rail wear

\begin{abstract}
A B S T R A C T
The prediction of the wear at the wheel-rail interface is a fundamental problem in the railway field, mainly correlated to the planning of maintenance interventions, vehicle stability and the possibility of researching specific strategies for the wheel and rail profile optimization. In this work the Authors present a model specifically developed for the evaluation of the wheel and rail profile evolution due to wear, whose layout is made up of two mutually interactive but separate units: a vehicle model for the dynamic analysis and a model for the wear estimation. The first one is made up of two parts that interact online during the dynamic simulations: a 3D multibody model of the railway vehicle implemented in Simpack Rail (a commercial software for the analysis of multibody systems) and an innovative 3D global contact model (developed by the Authors in previous works) for the detection of the contact points between wheel and rail and for the calculation of the forces in the contact patches (implemented in $\mathrm{C} / \mathrm{C}++$ environment). The wear model, implemented in the Matlab environment, is mainly based on experimental relationships found in literature between the removed material and the energy dissipated by friction at the contact. It starts from the outputs of the dynamic simulations (position of contact points, contact forces and global creepages) and calculates the pressures inside the contact patches through a local contact model (FASTSIM algorithm); then the material removed due to wear is evaluated and the worn profiles of wheel and rail are obtained. This approach allows the evaluation of both the quantity of removed material and its distribution along the wheel and rail profiles in order to analyze the development of the profiles shape during their lifetime.

The whole model is based on a discrete process: each discrete step consists in one dynamic simulation and one profile update by means of the wear model while, within the discrete step, the profiles are supposed to be constant. The choice of an appropriate step is fundamental in terms of precision and computational load. Moreover the different time scales characterizing the wheel and rail wear evolution require the development of a suitable strategy for the profile update: the strategy proposed by the Authors is based both on the total distance traveled by the considered vehicle and on the total tonnage burden on the track. The entire model has been developed and validated in collaboration with Trenitalia S.p.A. and Rete Ferroviaria Italiana (RFI), which have provided the technical documentation and the experimental results relating to some tests performed with the vehicle DMU Aln 501 Minuetto on the Aosta-Pre Saint Didier line.
\end{abstract}

(C) 2012 Elsevier B.V. All rights reserved.

\section{Introduction}

The wear at the wheel-rail interface is an important problem in the railway field. The evolution of the profile shape due to wear has a deep effect on the vehicle dynamics and on its running stability, leading to performance variations both in negotiating curves and in straight track. Therefore the original profiles have to be

\footnotetext{
* Corresponding author.

E-mail addresses: ignesti@mapp1.de.unifi.it (M. Ignesti), malvezzi@dii.unisi.it (M. Malvezzi), marini@mapp1.de.unifi.it (L. Marini), meli@mapp1.de.unifi.it (E. Meli), rindi@mapp1.de.unifi.it (A. Rindi).
}

periodically re-established by means of turning: particularly, from a safety viewpoint, the arising of a contact geometry which may compromise the vehicle stability or increase the derailment risk has to be avoided. A reliable wear model can also be used to optimize the original profiles of wheel and rail and to obtain a more uniform wear. In this way the overall amount of removed material can be reduced in order to increase the mean time between two maintenance intervals and, at the same time, the dynamical performance of the wheel-rail pair can be kept approximately constant between two turnings.

Because of all these reasons, the development of a mathematical model for the prediction of the wear at the wheel-rail interface represents a powerful tool. 


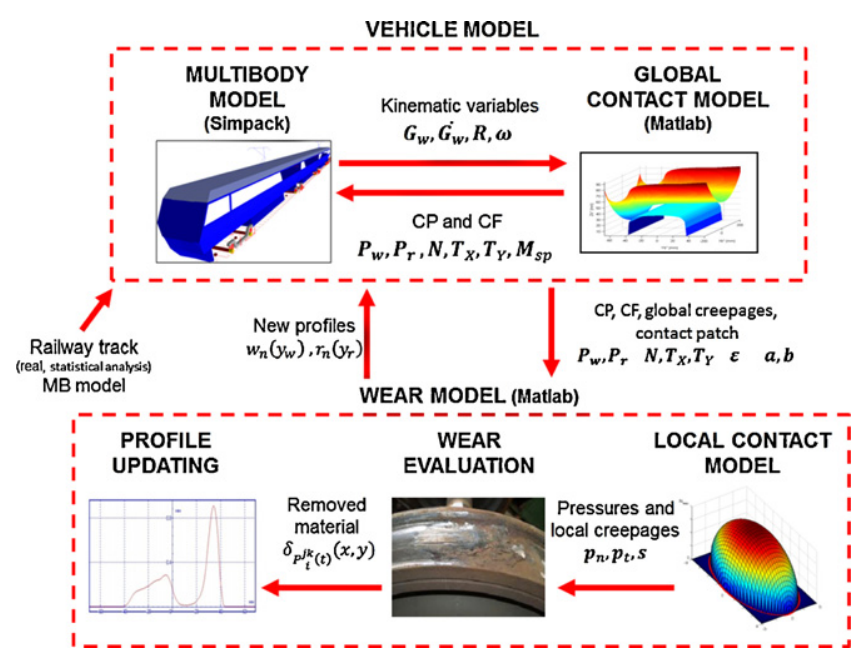

Fig. 1. General architecture of the model.

In this work the Authors will present a procedure to estimate the evolution of the wheel and rail profile due to wear based on a model that combines multibody and wear modeling. More specifically, the general layout of the model consists of two mutually interactive parts: the vehicle model (multibody model and 3D global contact model) and the wear model (local contact model and wear evaluation and profiles update). Concerning the vehicle model, the multibody model, implemented in the Simpack Rail environment, accurately reproduces the dynamics of the vehicle, taking into account all the significant degrees of freedom. The 3D global contact model, developed by the Authors in previous works [1,2], detects the wheel-rail contact points by means of an innovative algorithm based on suitable semi-analytic procedures and then, for each contact point, calculates the contact forces through Hertz's and Kalker's theory [3-5]. Thanks to the numerical efficiency of the new contact model, the two models interact directly online during the simulation of the vehicle dynamics.

As regards the wear estimation, the model is based on a local contact model (in this case the Kalker's FASTSIM algorithm) and on an experimental relationship for the calculation of the removed material $[6,7]$. The wear model, starting from the outputs of the vehicle model (contact points, contact forces and global creepages), calculates the total amount of removed material due to wear and its distribution along the wheel and rail profiles. The removal of the material is carried out considering the three dimensional structure of the contact bodies and the different time scales characterizing the wear evolution on the wheel and on the rail.

One of the most critical aspects in the development of a wear model is the availability of experimental data for the validation of the model, because the wear is a long-term phenomenon which requires several months of monitoring to collect the data. If on line experimental measurement cannot be carried out, the problem can be overcome using tools provided by software [8] or carrying out experimental proofs on a scaled test rig [6].

In this work the entire model has been validated by means of the experimental data provided by Trenitalia S.p.A. and RFI; the data concern the Aosta-Pre Saint Didier railway line and the vehicle ALSTOM DMU Aln 501 Minuetto which, in this scenery, exhibits serious problems in terms of wear.

\section{General architecture of the model}

The whole model consists of two different parts: the vehicle model and the wear model. The general architecture is shown in the block diagram in Fig. 1.
The vehicle model represents the dynamic analysis block and is composed by the multibody model of the studied railway vehicle (in this work the ALSTOM DMU Aln 501 Minuetto) and the 3D global contact model that, during the dynamic simulation, interact directly online creating a loop. At each time integration step the first one evaluates the kinematic variables (position, orientation and their derivatives) relative to the wheelset and consequently to each wheel - rail contact pair. Starting from these quantities, the second one calculates the global contact variables (contact points and contact forces, contact areas and global creepages). The 3D global contact model is based both on an innovative algorithm for the detection of the contact points (developed by the Authors in previous works $[1,2]$ ) and on Hertz's and Kalker's global theories for the evaluation of the contact forces [3]. The global contact variables are then passed to the multibody model in order to carry on the simulation of the vehicle dynamics.

The main inputs of the dynamic analysis block are the railway track and the multibody model of the considered vehicle: in this research activity, according to the specifications required by Trenitalia, the track is represented by a statistical analysis of the Aosta-Pre Saint Didier line by means of an ensemble of $N_{c}$ curvilinear tracks, each of length equal to $l_{\text {track }}$. The statistical analysis has been carried out splitting the considered track in radius classes and superelevation classes. The statistical approach has been introduced because of the complexity and the length of the Aosta-Pre Saint Didier track: for these reasons the simulation of the entire line would have caused difficulty both in terms of multibody modeling and in terms of numerical efficiency (computational load and memory consumption). The outputs of the vehicle model are the global contact variables evaluated during all the $N_{c}$ dynamic simulations and represent the inputs of the wear model.

The dynamic simulations have been performed in Simpack Rail. More specifically, the multibody model has been built using directly the Simpack Rail environment while the global contact model, implemented in $\mathrm{C} / \mathrm{C}++$, has been customized by means of the Simpack User Routine module (implemented in FORTRAN environment) that allows to handle the interaction between Simpack and routines defined by the user. The wear model is made up of three distinct phases: the local contact model, the wear evaluation and the profile update. Initially the local contact model (based both on Hertz's local theory and on simplified Kalker's algorithm FASTSIM), starting from the global contact variables, evaluates the local contact variables (contact pressures and local creepages) and divides the contact patch into adhesion area and creep area. Then, the distribution of removed material (hypothesizing the contact in dry conditions as required by Trenitalia and RFI) is calculated both on the wheel and on the rail surface only within the creep area using an experimental law between the removal material and the energy dissipated by friction at the contact interface [6,7]. Finally the profiles of wheel and rail are updated: the new profiles are the outputs of one discrete step of the whole model loop and have to be passed back to the vehicle model in order to continue the wear cycle described in Fig. 1 and to simulate the vehicle dynamics with updated profiles.

The evolution of the wheel and rail profiles is therefore a discrete process. In this research the choice of the discrete steps is one of the main issues and has to consider the difference of time scales between the wheel and rail wear evolution rate (as will be clarified in the following). For the wheel wear the following considerations are valid:

1. the total mileage $k m_{\text {tot }}$ traveled by the considered vehicle has been subdivided in constant steps of length equal to $\mathrm{km}_{\text {step }}$;

2. within each discrete step of the whole model (corresponding to $k m_{\text {step }}$ kilometers traveled by the vehicle) the wheel profile is supposed to be constant. 
Table 1

Inertia properties of the multibody model.

\begin{tabular}{lcccc}
\hline MBS body & Mass $(\mathrm{kg})$ & $\begin{array}{l}\text { Roll inertia } \\
\left(\mathrm{kg} \mathrm{m}^{2}\right)\end{array}$ & $\begin{array}{l}\text { Pitch inertia } \\
\left(\mathrm{kg} \mathrm{m}^{2}\right)\end{array}$ & $\begin{array}{l}\text { Yaw inertia } \\
\left(\mathrm{kg} \mathrm{m}^{2}\right)\end{array}$ \\
\hline Coach M & 31,568 & 66,700 & 764,000 & 743,000 \\
Coach T & 14,496 & 30,600 & 245,000 & 236,000 \\
Bogie m & 3306 & 1578 & 2772 & 4200 \\
Bogie t & 3122 & 1674 & 3453 & 5011 \\
Wheelset $\mathrm{m}$ & 2091 & 1073 & 120 & 1073 \\
Wheelset t & 1462 & 1027 & 120 & 1027 \\
\hline
\end{tabular}

The depth of the rail wear does not depend on the distance traveled by vehicle but on the number of vehicles moving on the track. Therefore a different approach for evaluating the discrete step for the rail, based on the total tonnage burden on the track $M_{t o t}$, is needed:

1. dividing the total tonnage $M_{\text {tot }}$ by the vehicle mass $M_{v}$, the corresponding vehicle number $N_{\text {tot }}$ has been calculated; then $N_{\text {tot }}$ has been subdivided in constant steps equal to $N_{\text {step }}$;

2 . within each discrete step (corresponding to $N_{\text {step }}$ vehicles moving on the track) the rail profile is supposed to be constant.

Finally the following considerations hold both for the wheel and the rail discretization steps:

- the number of discretization steps affects the model precision and the computational load. More particularly, increasing the step number, the model precision increases but, at the same time, the computational load increases too: a good compromise must be researched;

- various types of profile update strategies are available in literature $[9,10]$ : the constant step and the adaptive step strategies are the main ones. In the first one a constant update step is defined, while the second one is based on the definition of a threshold value that imposes the maximum material quantity to remove at each update of the profiles. The two methods have been compared and the first one has been chosen due to the following reasons:

1. the physical phenomenon of the wear evolution has usually an almost linear characteristic and thus is well suited to a constant update step;

2. the two methods lead to very similar system evolutions (both qualitatively and quantitatively) but the first one is computationally more efficient.

The wear model has been fully implemented in the Matlab environment.

\section{The vehicle model}

In this section a brief description of the vehicle model (composed by the multibody model and the global contact model) is given. In particular the global contact model will allow to the detection of the contact points between the wheel and rail and, subsequently, of the contact forces and the global creepages in the contact patch.

\subsection{The multibody model}

The DMU Aln 501 Minuetto, a passenger transport unit widely used on the Italian Railways, has been chosen as benchmark vehicle for this research; the physical and geometrical characteristics of the vehicle can be found in literature [11,12]. In Table 1 the inertia properties of the vehicle are shown: motors and gear boxes have not been modeled and their inertia properties have been included in the motor bogie and in the motor wheelset (indicated in Table 1

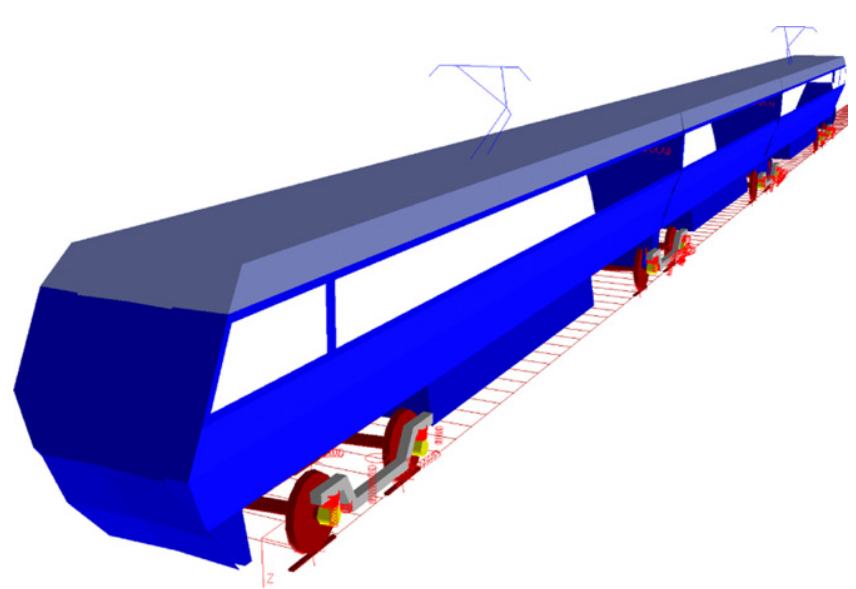

Fig. 2. Global view of the multibody model.

with Bogie $\mathbf{m}$ and Wheelset $\mathbf{m}$ respectively) in order to take into account their different influence on the unsprung and sprung mass. The multibody model has been realized in the Simpack Rail environment (see Fig. 2) and consists of thirty-one rigid bodies:

- three coaches;

- four bogies: the intermediate ones, interposed between two successive coaches, are trailer bogies while the other ones are motor bogies;

- eight wheelsets: two for each bogie;

- sixteen axleboxes: two for each wheelset.

The rigid bodies are connected by means of appropriate elastic and damping elements; particularly the vehicle is equipped with two suspension stages. The primary suspensions connect the wheelsets to the bogies (see Fig. 3) and comprise two springs and two vertical dampers, while the secondary suspensions connect the bogies to the coaches (see Fig. 4) and comprise the following elements:

- two air springs;

- six dampers (lateral, vertical and anti-yaw dampers);

- one traction rod;

- the roll bar (not visible in the figure);

- two lateral bumpstops.

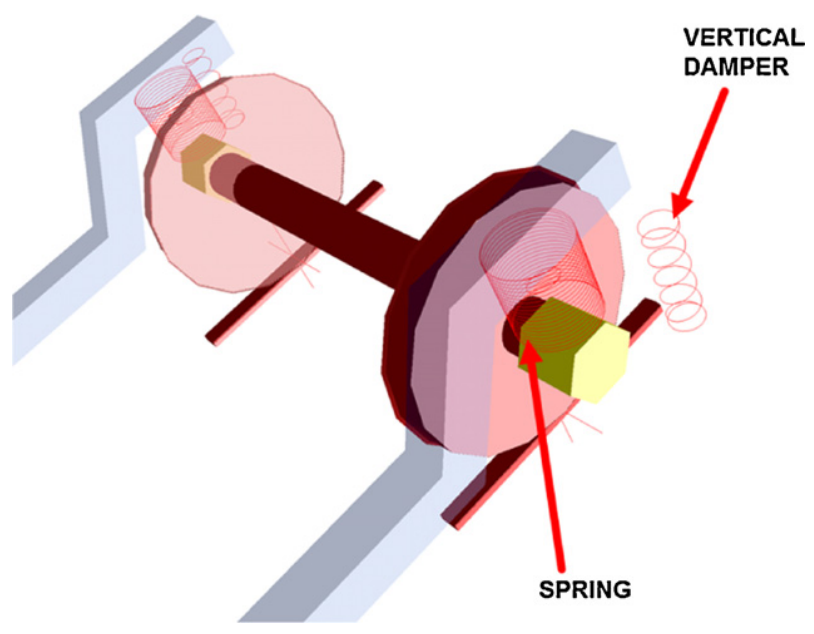

Fig. 3. Primary suspensions. 
Table 2

Main linear stiffness properties of the suspensions.

\begin{tabular}{|c|c|c|c|c|c|c|}
\hline MBS element & $\begin{array}{l}\text { Longitudinal stiffness } \\
(\mathrm{N} / \mathrm{m})\end{array}$ & $\begin{array}{l}\text { Lateral stiffness } \\
(\mathrm{N} / \mathrm{m})\end{array}$ & $\begin{array}{l}\text { Vertical stiffness } \\
(\mathrm{N} / \mathrm{m})\end{array}$ & $\begin{array}{l}\text { Roll stiffness } \\
(\mathrm{N} \mathrm{m} / \mathrm{rad})\end{array}$ & $\begin{array}{l}\text { Pitch stiffness } \\
(\mathrm{N} \mathrm{m} / \mathrm{rad})\end{array}$ & $\begin{array}{l}\text { Yaw stiffness } \\
(\mathrm{N} \mathrm{m} / \mathrm{rad})\end{array}$ \\
\hline $\begin{array}{l}\text { Primary } \\
\text { Suspension } \\
\text { Spring }\end{array}$ & $1,259,600$ & $1,259,600$ & 901,100 & 10,800 & 10,800 & 1000 \\
\hline $\begin{array}{l}\text { Secondary } \\
\text { Suspension } \\
\text { Air spring }\end{array}$ & 120,000 & 120,000 & 398,000 & - & - & - \\
\hline $\begin{array}{l}\text { Secondary } \\
\text { Suspension } \\
\text { Roll bar }\end{array}$ & - & - & $2,600,000$ & - & - & - \\
\hline
\end{tabular}

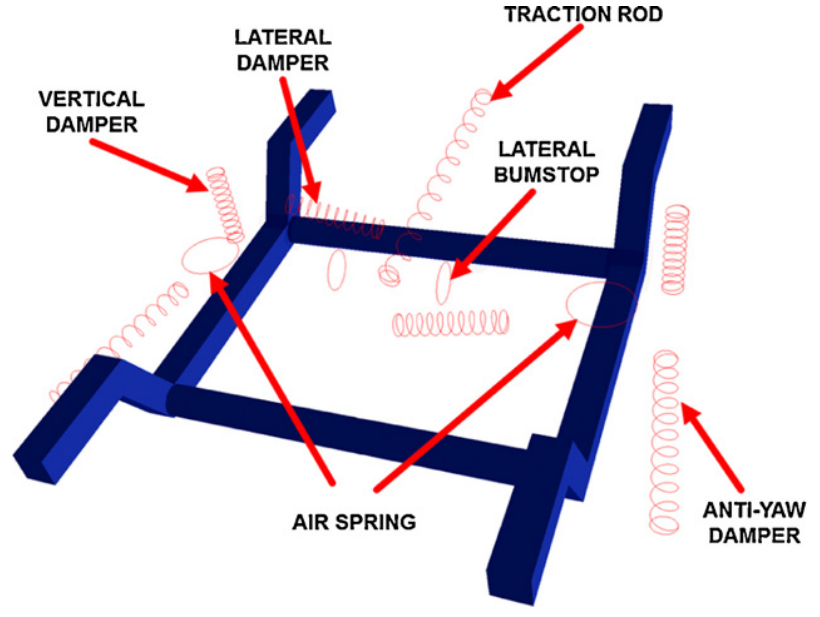

Fig. 4. Bogie and secondary suspensions.

Both the stages of suspensions have been modelled by means of three-dimensional viscoelastic force elements taking into account all the mechanical non linearities (bumpstop clearance, dampers and rod behavior). The main linear characteristics of the suspensions are shown in Table 2 while the nonlinear characteristics are imposed as a function of displacement and velocity for the springs and the dampers respectively (see Figs. 5 and 6).

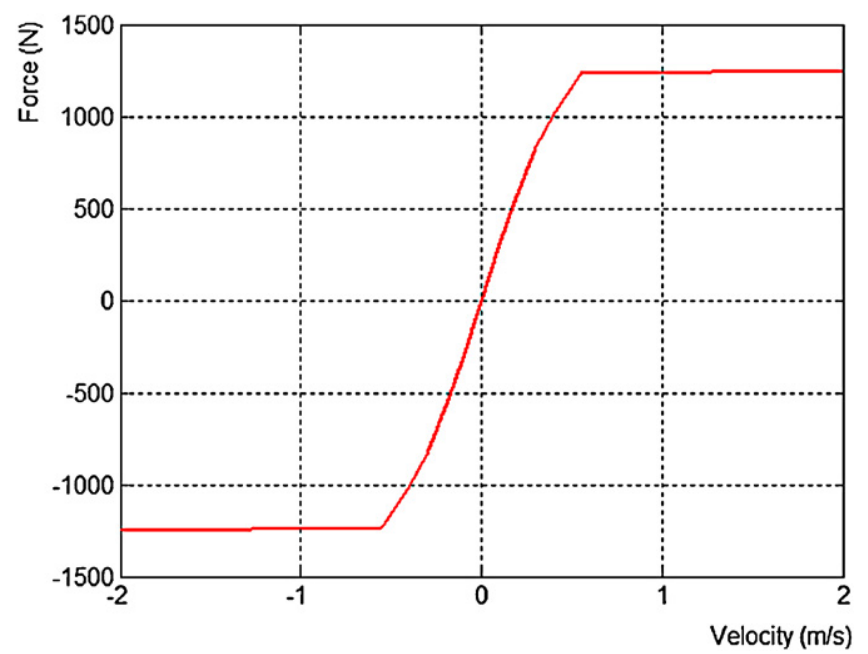

Fig. 5. Example of nonlinear characteristic: vertical damping of the primary suspension.

\subsection{The global contact model}

Dynamic simulations of railway vehicles need a reliable and efficient method to evaluate the contact points between wheel and rail, because their position has a considerable influence both on the direction and on the magnitude of the contact forces. In this work a specific contact model has been considered instead of that implemented in Simpack Rail in order to achieve better reliability and accuracy [1,2]. The proposed contact model is divided in two parts: in the first one the contact points are detected by means of an innovative algorithm developed by the Authors in previous works $[1,2]$, while in the second one the global contact forces acting at the wheel-rail interface are evaluated by means of Hertz's and Kalker's global theories [3-5].

The algorithm for the contact points detection starts from the standard idea that the contact points make stationary the distance between the wheel and rail surfaces (see Fig. 7(a)); in more details the distance has a point of relative minimum if there is no penetration between the considered surfaces, while it has a relative maximum in the other case (see Section 3.2.1 for the analytic formulation of the problem). The main features of the innovative adopted algorithm are the following:

- it is a fully 3D model that takes into account all the six relative degrees of freedom (DOF) between wheel and rail;

- it is able to support generic railway tracks and generic wheel and rail profiles;

- it assures a general and accurate treatment of the multiple contact without introducing simplifying assumptions on the problem geometry and limits on the number of contact points detected;

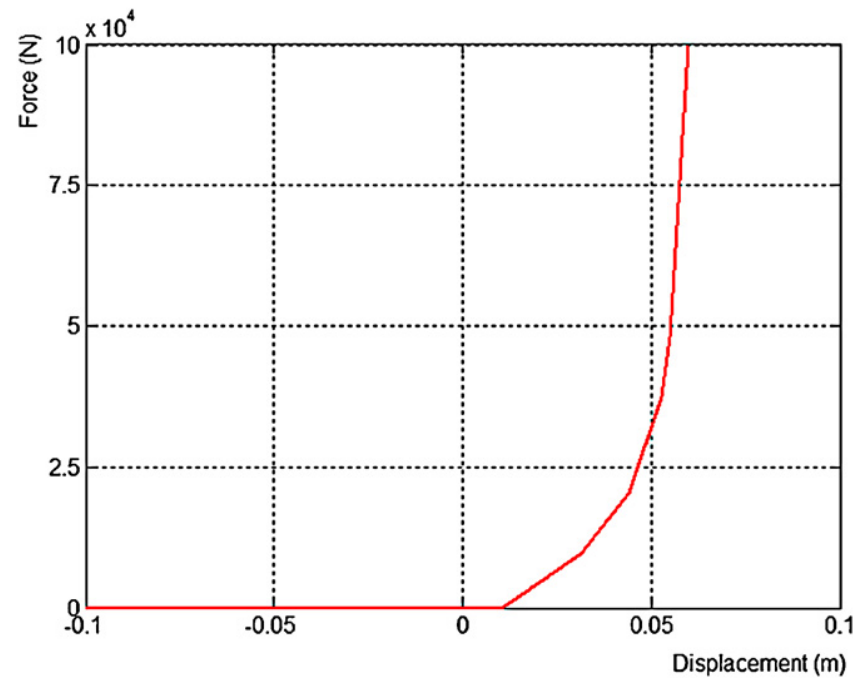

Fig. 6. Example of nonlinear characteristic: lateral bumpstop stiffness of the secondary suspension. 
(a)

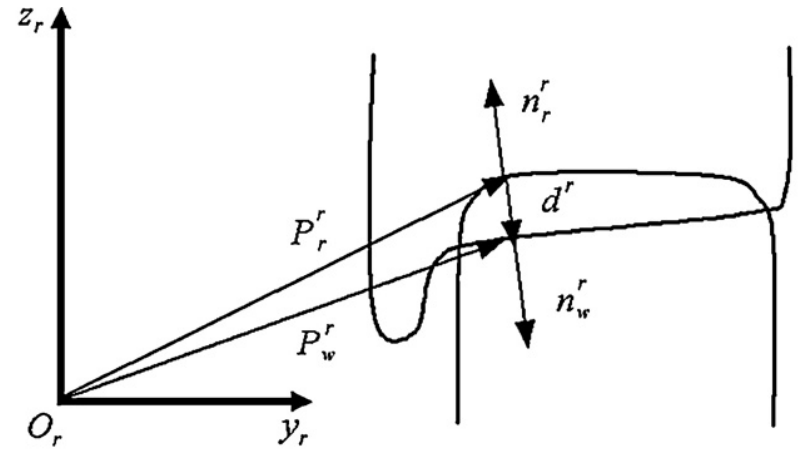

(b)

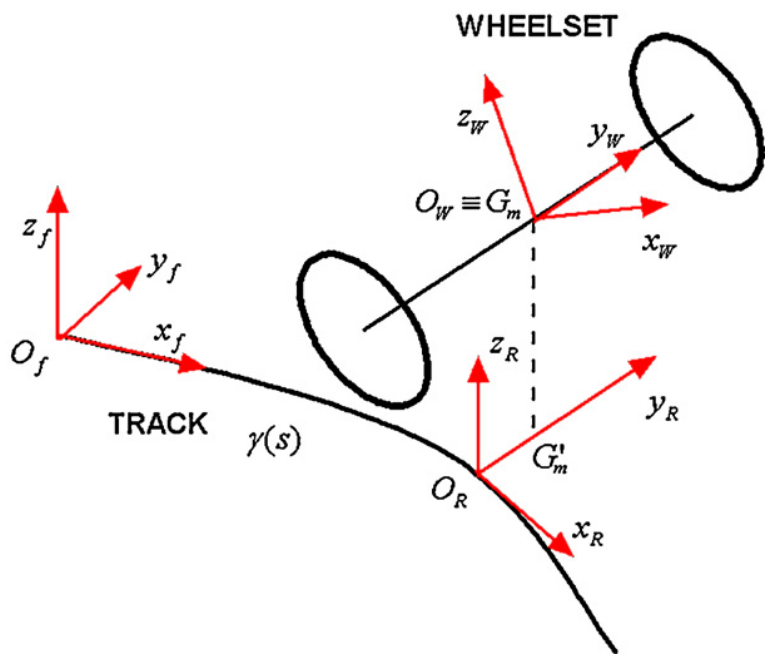

Fig. 7. Distance method.

- it assures highly numerical efficiency making possible the online implementation within the commercial multibody software (Simpack-Rail, Adams-Rail); in this way also the numerical performance of the commercial multibody software are improved.

Two specific reference systems have to be introduced in order to simplify the model's equations: the auxiliary reference system and the local reference system. The auxiliary system $O_{r} x_{r} y_{r} z_{r}$ is defined on the plane of the rails and follows the motion of the wheelset during the dynamic simulations: the $x_{r}$ axis is tangent to the center line of the track in the origin $O_{r}$, whose position is defined so that the $y_{r} z_{r}$ plane contains the center of mass $\mathbf{G}_{w}$ of the wheelset, and the $z_{r}$ axis is perpendicular to plane of the rails. The local system $O_{w} x_{w} y_{w} z_{w}$ is rigidly connected to the wheelset except for the rotation around its axis and the $x_{w}$ axis is parallel to the $x_{r} y_{r}$ plane (see Fig. 7(b)). In the following, for the sake of simplicity, the variables referred to the local system will be marked with the apex $w$, while those referred to the auxiliary system with the apex $r$; the variables belonging to the wheel and to the rail will be indicated with the subscripts $w$ and $r$ respectively.

Thanks to these reference systems, the definition of the wheel and rail geometrical surfaces is easy. In the local reference system the wheelset can be considered as a simple revolution surface around its axis $y_{w}$ (see Eq. (2)); the generating function, indicated by $w\left(y_{w}\right)$, is supposed to be known (in this work, the profile ORE $S$ 1002 for a single wheel has been chosen (see Fig. 8)). Similarly the track can be locally described in the auxiliary reference system as an estrusion surface along the $x_{r}$ axis (see Eq. (3)); the generating function, indicated by $r\left(y_{r}\right)$, is known (the profile UIC 60, with laying angle $\alpha_{p}$ equal to $1 / 20$ rad, has been chosen for a single rail (see Fig. 8)).
With reference to Fig. 1, the global contact model can be thought of as a black box having the following inputs and outputs:

- Inputs: the kinematic variables evaluated by the multibody model, i.e. the position $\mathbf{O}_{w}^{r}$, the orientation matrix $R_{w}^{r}$, the absolute velocity $\dot{\mathbf{O}}_{w}^{r}$ and the absolute angular velocity $\boldsymbol{\omega}_{w}^{r}$ of the wheel system and the analogous quantities of the rail system $\mathbf{O}_{r}^{r}=\mathbf{0}, R_{r}^{r}=I$, $\dot{\mathbf{O}}_{r}$ and $\omega_{r}^{r}$;

- Outputs: the global contact variables relative to the wheel-rail interface, like the positions $\mathbf{P}_{w}^{r}$ and $\mathbf{P}_{r}^{r}$ of the contact points, the contact forces (the normal component $N^{r}$ and the tangential components $T_{x}^{r}$ and $T_{y}^{r}$ ), the global creepages $\varepsilon_{x}, \varepsilon_{y}$ and $\varepsilon_{s p}$ and the contact patch's dimensions $a, b$.

\subsubsection{The distance method algorithm}

In this subsection the algorithm used for detecting the contact points will be described. The distance method algorithm (see Fig. 7(a)) is based on a classical formulation of the contact problem in multibody field:

$\mathbf{n}_{r}^{r}\left(\mathbf{P}_{r}^{r}\right) \wedge \mathbf{n}_{w}^{r}\left(\mathbf{P}_{w}^{r}\right)=\mathbf{n}_{r}^{r}\left(\mathbf{P}_{r}^{r}\right) \wedge R_{w}^{r} \mathbf{n}_{w}^{w}\left(\mathbf{P}_{w}^{w}\right)=\mathbf{0}$

$\mathbf{n}_{r}^{r}\left(\mathbf{P}_{r}^{r}\right) \wedge \mathbf{d}^{r}=\mathbf{0}$

where:

- $\mathbf{P}_{w}^{w}$ and $\mathbf{P}_{r}^{r}$ are the positions of the generic point on the wheel surface and on the rail surface expressed in their reference systems:

$$
\begin{aligned}
& \mathbf{P}_{w}^{w}\left(x_{w}, y_{w}\right)=\left(\begin{array}{lll}
x_{w} & y_{w} & -\sqrt{w\left(y_{w}\right)^{2}-x_{w}^{2}}
\end{array}\right)^{T} \\
& \mathbf{P}_{r}^{r}\left(x_{r}, y_{r}\right)=\left(\begin{array}{lll}
x_{r} & y_{r} & r\left(y_{r}\right)
\end{array}\right)^{T}
\end{aligned}
$$

- $\mathbf{n}_{w}^{w}$ and $\mathbf{n}_{r}^{r}$ are the outgoing normal unit vectors to the wheel and rail surface respectively and are defined as follows:

$$
\mathbf{n}_{w}^{w}\left(\mathbf{P}_{w}^{w}\right)=\frac{-\frac{\partial \mathbf{P}_{w}^{w}}{\partial x_{w}} \wedge \frac{\partial \mathbf{P}_{w}^{w}}{\partial y_{w}}}{\left\|\frac{\partial \mathbf{P}_{w}^{w}}{\partial x_{w}} \wedge \frac{\partial \mathbf{P}_{w}^{w}}{\partial y_{w}}\right\|} \quad, \quad \mathbf{n}_{r}^{r}\left(\mathbf{P}_{r}^{r}\right)=\frac{\frac{\partial \mathbf{P}_{r}^{r}}{\partial x_{r}} \wedge \frac{\partial \mathbf{P}_{r}^{r}}{\partial y_{r}}}{\left\|\frac{\partial \mathbf{P}_{r}^{r}}{\partial x_{r}} \wedge \frac{\partial \mathbf{P}_{r}^{r}}{\partial y_{r}}\right\|}
$$

- $R_{w}^{r}$ is the rotation matrix that links the local reference system to the auxiliary one;

- $\mathbf{d}^{r}$ is the distance vector between two generic points on the wheel surface and on the rail surface (both referred to the auxiliary system) and it is equal to:

$\mathbf{d}^{r}\left(x_{w}, y_{w}, x_{r}, y_{r}\right)=\mathbf{P}_{w}^{r}\left(x_{w}, y_{w}\right)-\mathbf{P}_{r}^{r}\left(x_{r}, y_{r}\right)$

where $\mathbf{P}_{w}^{r}$ is the position of the generic point of the wheel surface expressed in the auxiliary system:

$\mathbf{P}_{w}^{r}\left(x_{w}, y_{w}\right)=\mathbf{0}_{w}^{r}+R_{w}^{r} \mathbf{P}_{w}^{w}\left(x_{w}, y_{w}\right)$.

The first condition (Eq. (1a)) of the system (1) imposes the parallelism between the normal unit vectors, while the second one (Eq. (1b)) requires the parallelism between the normal unit vector to the rail surface and the distance vector.

Alternatively, other classical formulations of the contact problem can be used, by example imposing the orthogonality between the $\mathbf{d}^{r}$ vector and the tangential planes to the wheel and rail surfaces (respectively $\pi_{w}$ and $\pi_{r}$ ); however this approach leads to more complex and less manageable calculations and for this reason has not be adopted.

The system (1) consists of six nonlinear equations in the unknowns $\left(x_{w}, y_{w}, x_{r}, y_{r}\right)$ (only four equations are independent and therefore the problem is $4 \mathrm{D})$. However it is possible to express three 

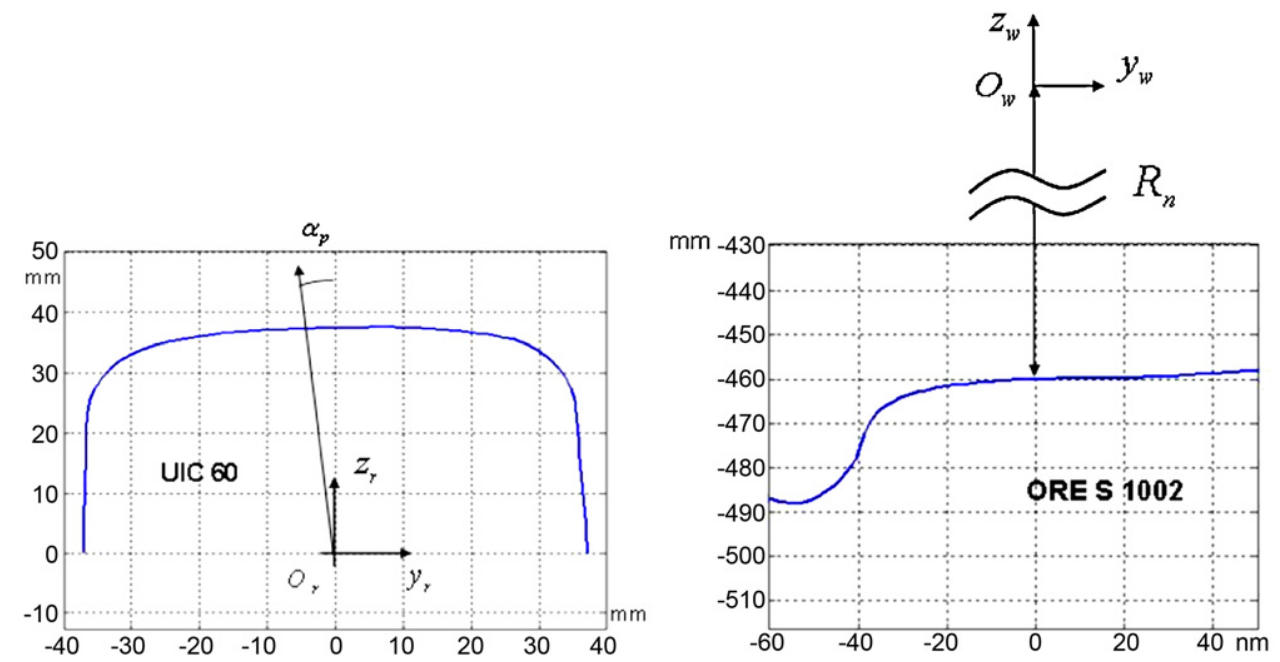

Fig. 8. Wheel and rail profiles.

of the four variables (in this case $\left.\left(x_{w}, x_{r}, y_{r}\right)\right)$ as a function of $y_{w}$, reducing the original $4 \mathrm{D}$ problem to a simple $1 \mathrm{D}$ scalar equation.

The reduction of the problem dimension using appropriate analytical procedures is the most innovative aspect of the proposed method. The second component of Eq. (1a) leads to the following equation:

$r_{13} \sqrt{w\left(y_{w}\right)^{2}-x_{w}^{2}}=r_{11} x_{w}-r_{12} w\left(y_{w}\right) w^{\prime}\left(y_{w}\right)$

where $r_{i j}$ are the elements of the $R_{w}^{r}$ matrix. Calling $A=r_{13}$, $B=w\left(y_{w}\right), C=r_{11}$ and $D=r_{12} w\left(y_{w}\right) w^{\prime}\left(y_{w}\right)$, the previous equation becomes:

$A \sqrt{B^{2}-x_{w}^{2}}=C x_{w}-D$

Hence, removing the radical and solving for $x_{w}$ :

$x_{w 1,2}\left(y_{w}\right)=\frac{C D \pm \sqrt{C^{2} D^{2}-\left(A^{2}+C^{2}\right)\left(D^{2}-A^{2} B^{2}\right)}}{A^{2}+C^{2}} ;$

as can be seen, there are two possible values of $x_{w}$ for each $y_{w}$.

From the first component of Eq. (1a) the following relation for $r^{\prime}\left(y_{r}\right)$ holds:

$$
\begin{aligned}
& r^{\prime}\left(y_{r}\right)_{1,2} \\
& =\frac{r_{21} x_{r 1,2}\left(y_{w}\right)-r_{22} w\left(y_{w}\right) w^{\prime}\left(y_{w}\right)-r_{23} \sqrt{w\left(y_{w}\right)^{2}-x_{w 1,2}\left(y_{w}\right)^{2}}}{r_{32} w\left(y_{w}\right) w^{\prime}\left(y_{w}\right)+r_{33} \sqrt{w\left(y_{w}\right)^{2}-x_{w 1,2}\left(y_{w}\right)^{2}}} .
\end{aligned}
$$

If $r^{\prime}\left(y_{r}\right)_{1,2}$ is a decreasing monotonous function (considering separately the sides of the track), Eq. (10) is numerically invertible and a single pair $y_{r 1,2}\left(y_{w}\right)$ exists for each $y_{w}$ value; otherwise the numerical inversion is still possible but will produce a further multiplication of the solution number.

By the second component of Eq. (1b) the expression of $x_{r 1,2}\left(y_{w}\right)$ can be obtained:

$x_{r 1,2}\left(y_{w}\right)=r_{11} x_{w 1,2}\left(y_{w}\right)+r_{12} y_{w}-r_{13} \sqrt{w\left(y_{w}\right)^{2}-x_{w 1,2}\left(y_{w}\right)^{2}}$.
Finally, replacing the variables $x_{w 1,2}\left(y_{w}\right), x_{r 1,2}\left(y_{w}\right)$ and $y_{r 1,2}\left(y_{w}\right)$ in the first component of Eq. (1b), the following 1D scalar equation can be written:

$$
\begin{aligned}
F_{1,2}\left(y_{w}\right)= & -r^{\prime}\left[G_{m z}+r_{32} y_{w}-r_{33} \sqrt{w^{2}-x_{w 1,2^{2}}}-b\right]+ \\
& -\left[G_{m y}+r_{21} x_{w 1,2}+r_{22} y_{w}-r_{23} \sqrt{w^{2}-x_{w 1,2^{2}}}\right. \\
& \left.-y_{r 1,2}\right]=0
\end{aligned}
$$

where $G_{w x}, G_{w y}, G_{w z}$ are the coordinates of the wheelset center of mass in the auxiliary system. The expression (12) consists of two scalar equations in the variable $y_{w}$ that can be easily solved with appropriate numerical algorithms.

The advantages of this approach based on the reduction of the algebraic problem dimension are many and can be summarized as follows:

- the reduction of the algebraic problem dimension from 4D to 1D allows to obtain an high numerical efficiency that makes possible the online implementation of the new method within the multibody vehicle models;

- the analytical approach assures an high degree of accuracy and generality;

- the 1D problem assures an easier management of the multiple solutions from an algebraic and a numerical point of view;

- in $1 \mathrm{D}$ problem also particularly elementary numerical algorithms like the grid method are quite efficient.

Thus, once obtained the generic solution (indicated with the subscript i) $y_{w i}$ of Eq. (12), the complete solution $\left(x_{w i}, y_{w i}, x_{r i}\right.$, $y_{r i}$ ) of the system (1) and consequently the contact points $\mathbf{P}_{w i}^{r}=$ $\mathbf{P}_{w}^{r}\left(x_{w i}, y_{w i}\right)$ and $\mathbf{P}_{r i}^{r}=\mathbf{P}_{r}^{r}\left(x_{r i}, y_{r i}\right)$ can be found by substitution.

However, since Eqs. (1a) and (1b) contain irrational terms, the generic solution $\left(x_{w i}, y_{w i}, x_{r i}, y_{r i}\right)$ must satisfy the following analytical conditions:

- the solution must be real;

- the solution does not have to generate complex terms (that could be caused by the radicals in the equations);

- the solution must be an effective solution of the system (1) (check necessary because of the radical removal by squaring).

Furthermore, from a physical point of view, also the next checks must be evaluated: 


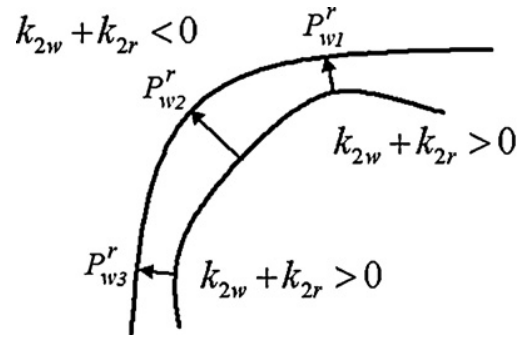

Fig. 9. Convexity conditions.

- the multiple solutions obtained from the analytical resolution of Eq. (12) must be individuated and erased because they have no physical meaning;

- the following convexity conditions must be satisfied so that the contact is physically possible:

$k_{1, w i}+k_{1, r i}>0$
$k_{2, w i}+k_{2, r i}>0$

where $k_{1, w i}, k_{2, w i}$ are the normal curvature of the wheel surface in longitudinal and lateral direction (referred to the auxiliary system and evaluated in the $i$ th contact point $\left.\left(x_{w i}, y_{w i}, x_{r i}, y_{r i}\right)\right)$ while $k_{1, r i}$, $k_{2, r i}$ are the analogous quantities for the rail surface. Because of the problem geometry, the first one of Eq. (13) is always satisfied and thus only the second one must be verified (see Fig. 9).

- the generic solution of the system (1) can be an effective contact point only if the normal penetration $\tilde{p}_{n}$ between the surfaces of wheel and rail is negative (according to the adopted convention), i.e. there must be effective penetration between the bodies:

$\tilde{p}_{n i}=\mathbf{d}_{i}^{r} \cdot \mathbf{n}_{r}^{r}\left(\mathbf{P}_{r i}^{r}\right)=-\mathbf{d}_{i}^{r} \cdot \mathbf{n}_{w}^{r}\left(\mathbf{P}_{w i}^{r}\right)<0$.

\subsubsection{The contact forces}

Then, for each contact point, the normal and tangential contact forces and the global creepages on the contact patch are determined (see Fig. 10).

The normal forces $N^{r}$ (expressed in the auxiliary system) are calculated by means of Hertz's theory [5,13]:

$N^{r}=\left[-k_{h}\left|\tilde{p}_{n}\right|^{\gamma}+k_{v}\left|v_{n}\right| \frac{\operatorname{sign}\left(v_{n}\right)-1}{2}\right] \frac{\operatorname{sign}\left(\tilde{p}_{n}\right)-1}{2}$

where:

- $\tilde{p}_{n}$ is the normal penetration defined by Eq. (14);

- $\gamma$ is the Hertz's exponent equal to $3 / 2$;

- $k_{v}$ is the contact damping constant $\left(k_{v}=10^{5} \mathrm{~N} \mathrm{~s} / \mathrm{m}\right)$;

- $v_{n}=\mathbf{V} \cdot n_{r}^{r}$ is the normal penetration velocity ( $\mathbf{V}$ is the velocity of the contact point rigidly connected to the wheelset);

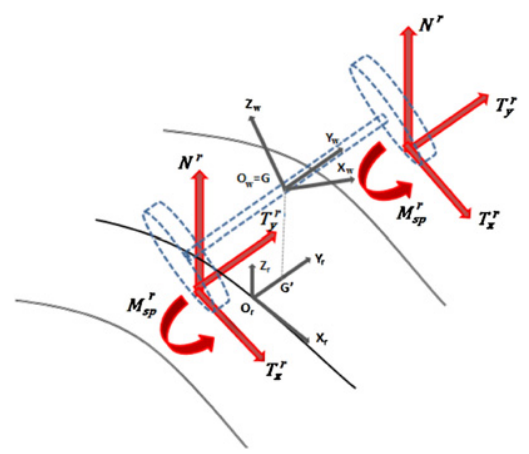

Fig. 10. Global forces acting at wheel and rail interface.
- $k_{h}$ is the hertzian constant, function both of the material properties and of the geometry of the contact bodies (curvatures and semiaxes of the contact patch) [14,2].

The global creepages $\boldsymbol{\varepsilon}$ (longitudinal $\varepsilon_{x}$, lateral $\varepsilon_{y}$ and spin creepage $\left.\varepsilon_{s p}\right)$ are calculated as follows:

$\varepsilon_{x}=\frac{\mathbf{V} \cdot \mathbf{i}_{r}}{\left\|\dot{\mathrm{O}}_{w}^{r}\right\|} \quad, \quad \varepsilon_{y}=\frac{\mathbf{V} \cdot \mathbf{t}_{r}^{r}\left(\mathbf{P}_{r}^{r}\right)}{\left\|\dot{\mathrm{O}}_{w}^{r}\right\|} \quad, \quad \varepsilon_{s p}=\frac{\boldsymbol{\omega}_{w}^{r} \cdot \mathbf{n}_{r}^{r}\left(\mathbf{P}_{r}^{r}\right)}{\left\|\dot{\mathrm{O}}_{w}^{r}\right\|}$

where $\mathbf{V}$ is the velocity of contact point rigidly connected to the wheelset, $\dot{\mathbf{O}}_{w}^{r}$ is the wheelset center of mass velocity (taken as the reference velocity for the calculation of the global creepages), $\omega_{w}^{r}$ is the angular velocity of wheelset expressed in auxiliary system, $\mathbf{i}_{r}$ is the unit vector in longitudinal direction of the auxiliary system and $\mathbf{t}_{r}^{r}$ is the tangential unit vector to the rail profile.

The tangential contact forces $\tilde{T}_{x}^{r}, \tilde{T}_{y}^{r}$ and the spin torque $M_{s p}^{r}$ (expressed in the auxiliary system) are calculated by means of the Kalker's global theory:

$\tilde{T}_{x}^{r}=-f_{11} \varepsilon_{x}, \quad \tilde{T}_{y}^{r}=-f_{22} \varepsilon_{y}-f_{23} \varepsilon_{s p}$

$M_{s p}^{r}=f_{23} \varepsilon_{y}-f_{33} \varepsilon_{s p}$

where the coefficients $f_{i j}$ are function both of the materials and of the semiaxes of the contact patch:

$f_{11}=a b G C_{11}, \quad f_{22}=a b G C_{22}$

$f_{23}=(a b)^{3 / 2} G C_{23}, \quad f_{33}=(a b)^{2} G C_{33}$

in which $G$ is the wheel and rail combined shear modulus and $C_{i j}$ are the Kalker's coefficients that can be found tabulated in literature [3]. At this point, it is necessary to introduce a saturation on the tangential contact forces $\tilde{T}^{r}=\left[\begin{array}{ll}\tilde{T}_{x}^{r} & \tilde{T}_{y}^{r}\end{array}\right]^{T}$, in order to consider the adhesion limit (not taken in account by the linear Kalker's theory):

$\left\|\mathbf{T}^{r}\right\| \leq \mu_{c} N^{r}$

where $\mu_{c}$ is the kinetic friction coefficient. Consequently the saturated tangential forces $\mathbf{T}^{r}$ will have the following expression:

$\mathbf{T}^{r}=\epsilon \tilde{\mathrm{T}}^{\mathrm{r}}$

in which the saturation coefficient $\epsilon$ can be evaluated as follows $[15,16]$ :

$\epsilon= \begin{cases}\frac{\mu_{c} N^{r}}{\tilde{T}^{r}}\left[\left(\frac{\tilde{T}^{r}}{\mu_{c} N^{r}}\right)-\frac{1}{3}\left(\frac{\tilde{T}^{r}}{\mu_{c} N^{r}}\right)^{2}+\frac{1}{27}\left(\frac{\tilde{T}^{r}}{\mu_{c} N^{r}}\right)^{3}\right] & \text { if } \tilde{T}^{r} \leq 3 \mu_{c} N^{r} \\ \frac{\mu_{c} N^{r}}{\tilde{T}^{r}} & \text { if } \tilde{T}^{r}>3 \mu_{c} N^{r}\end{cases}$

where $\tilde{T}_{r}=\left\|\tilde{\mathrm{T}}^{r}\right\|$.

\section{The wear model}

In this section the three phases, in which the wear model has been divided, will be described in details: the local contact model, the evaluation of the amount of removed material (assuming dry contact conditions) and the wheel and rail profile update.

\subsection{The local contact model}

The purpose of the local contact model is the calculation of the local contact variables (normal and tangential contact stresses $p_{n}$, $\mathbf{p}_{t}$ and local creep $\mathbf{s}$, all evaluated within the contact patch) starting from the corresponding global variables (contact points $\mathbf{P}_{w}^{r}, \mathbf{P}_{r}^{r}$, contact forces $N^{r}, T_{x}^{r}, T_{y}^{r}$, global creepage $\boldsymbol{\varepsilon}$ and semiaxes of the contact patch $a, b)$. 


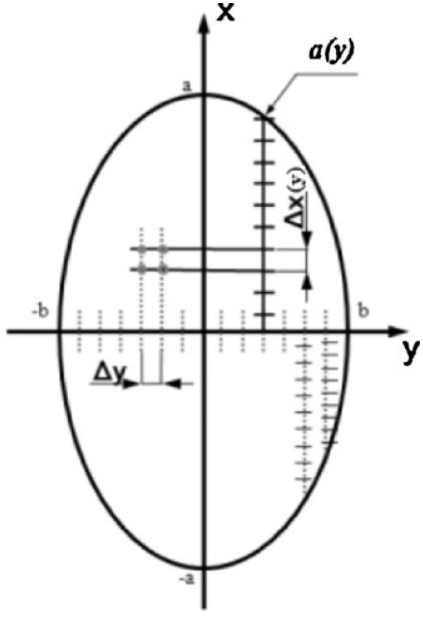

Fig. 11. Contact patch discretization.

This model is based on the Kalker's local theory in the simplified version implemented in the algorithm FASTSIM; this algorithm contains an extremely efficient version (although necessarily approximate) of the Kalker theory and therefore is widely used in railway field [17].

For the local analysis a new reference system is defined at the wheel-rail interface on the contact plane (i.e. the common tangent plane between the wheel and rail surfaces): the $x$ and $y$ axes are the longitudinal and the transversal direction of the contact plane respectively (see Figs. 11 and 13). The algorithm is based on the proportionality hypothesis between the tangential contact pressure $\mathbf{p}_{t}$ and the elastic displacements $\mathbf{u}$, both evaluated within the contact patch:

$\mathbf{u}(x, y)=L \mathbf{p}_{t}(x, y), \quad L=L(\varepsilon, a, b, G, v)$

where the flexibility $L$ (function of the global creepages $\varepsilon$, the semiaxes of the contact patch $a, b$, the wheel and rail combined shear modulus $G$ and the wheel and rail combined Poisson's ratio $v$ ) can be calculated as follows:

$L=\frac{\left|\varepsilon_{x}\right| L_{1}+\left|\varepsilon_{y}\right| L_{2}+c\left|\varepsilon_{s p}\right| L_{3}}{\left(\varepsilon_{x}^{2}+\varepsilon_{y}^{2}+c^{2} \varepsilon_{s p}^{2}\right)^{1 / 2}}$

with $L_{1}=8 a /\left(3 G C_{11}\right), L_{2}=8 a /\left(3 G C_{22}\right), L_{3}=\pi a^{2} /\left(4 G c C_{23}\right)$ and $c=\sqrt{a b}$ (the constants $C_{i j}$, functions both of the Poisson's ratio $v$ and of the ratio $a / b$, are the Kalker's parameters and can be found in literature [17]).

The local creepages $\mathbf{s}$ can be calculated by derivation considering both the elastic creepages and the rigid ones:

$\mathbf{s}(x, y)=\dot{\mathbf{u}}(x, y)+V\left(\begin{array}{c}\varepsilon_{x} \\ \varepsilon_{y}\end{array}\right)$

where $V=\left\|\dot{\mathbf{o}}_{w}^{r}\right\|$ is the longitudinal vehicle speed. At this point it is necessary to discretize the elliptical contact patch in a grid of points in which the quantities $p_{n}, \mathbf{p}_{t}$ and $\mathbf{s}$ will be evaluated. Initially the transversal axis (with respect to the motion direction) of the contact ellipse has been divided in $n_{y}-1$ equal parts of magnitude $\Delta y=2 b /\left(n_{y}-1\right)$ by means of $n_{y}$ equidistant nodes. Then the longitudinal sections of the patch (long $2 a(y)=2 a \sqrt{1-y / b^{2}}$ ) have been divided in $n_{x}-1$ equal parts of magnitude $\Delta x(y)=2 a(y) /\left(n_{x}-1\right)$ by means of $n_{x}$ equidistant nodes (see Fig. 11). Due to this strategy the longitudinal grid resolution is not constant but increases near the lateral edges of the ellipse, where the lengths $a(y)$ are smaller. This procedure provides more accurate results right next to the edges of the ellipse, where a constant resolution grid would generate excessive numerical noise. The values of the $n_{x}$ and $n_{y}$ parameters have to assure the right balance between precision and computational load; good values of compromise are in the range [25 50].

Once the contact patch is discretized, the FASTSIM algorithm allows the iterative evaluation of both the contact pressures value $p_{n}, \mathbf{p}_{t}$ and the local creepage $\mathbf{s}$ in order to divide the contact patch in adhesion and slip zone. Indicating the generic point of the grid with $\left(x_{i}, y_{j}\right), 1 \leq i \leq n_{x} 1 \leq j \leq n_{y}$, the normal contact pressure can be expressed as:

$p_{n}\left(x_{i}, y_{j}\right)=\frac{3}{2} \frac{N^{r}}{\pi a b} \sqrt{1-\frac{x_{i}^{2}}{a^{2}}-\frac{y_{j}^{2}}{b^{2}}}$

where $N^{r}$ is the normal contact force, while the limit adhesion pressure $\mathbf{p}_{A}$ is:

$\mathbf{p}_{A}\left(x_{i}, y_{j}\right)=\mathbf{p}_{t}\left(x_{i-1}, y_{j}\right)-\left(\begin{array}{c}\varepsilon_{x} \\ \varepsilon_{y}\end{array}\right) \frac{\Delta x\left(y_{j}\right)}{L} ;$

thus, knowing the variable values in the point $\left(x_{i-1}, y_{j}\right)$, it is possible to pass to the point $\left(x_{i}, y_{j}\right)$ as follows:

$$
\begin{aligned}
& \text { if }\left\|\mathbf{p}_{A}\left(x_{i}, y_{j}\right)\right\| \leq \mu p_{n}\left(x_{i}, y_{j}\right) \\
& \text { arrow } \\
&\left\{\begin{array}{l}
\mathbf{p}_{t}\left(x_{i}, y_{j}\right)=\mathbf{p}_{A}\left(x_{i}, y_{j}\right) \\
\mathbf{s}\left(x_{i}, y_{j}\right)=\mathbf{0}
\end{array}\right. \\
& \text { if }\left\|\mathbf{p}_{A}\left(x_{i}, y_{j}\right)\right\|>\mu p_{n}\left(x_{i}, y_{j}\right) \\
&\left\{\begin{array}{l}
\mathbf{p}_{t}\left(x_{i}, y_{j}\right)=\mu p_{n}\left(x_{i}, y_{j}\right) \mathbf{p}_{A}\left(x_{i}, y_{j}\right) /\left\|\mathbf{p}_{A}\left(x_{i}, y_{j}\right)\right\| \\
\mathbf{s}\left(x_{i}, y_{j}\right)=\frac{L V}{\Delta x\left(y_{j}\right)}\left(\mathbf{p}_{t}\left(x_{i}, y_{j}\right)-\mathbf{p}_{A}\left(x_{i}, y_{j}\right)\right)
\end{array}\right.
\end{aligned}
$$

where $\mu$ is the static friction coefficient; Eqs. (28a) and (28b) hold respectively in the adhesion and slip zone. Iterating the procedure for $2 \leq i \leq n_{x}$ and successively for $1 \leq j \leq n_{y}$ and assuming as boundary conditions $\mathbf{p}_{t}\left(x_{1}, y_{j}\right)=\mathbf{0}, \mathbf{s}\left(x_{1}, y_{j}\right)=0$ for $1 \leq j \leq n_{y}$ (i.e. stresses and creepages zero out of the contact patch), the desired distribution of $p_{n}\left(x_{i}, y_{j}\right), \mathbf{p}_{t}\left(x_{i}, y_{j}\right)$ and $\mathbf{s}\left(x_{i}, y_{j}\right)$ can be determined.

\subsection{The wear evaluation}

To evaluate the distribution of removed material on wheel and rail due to wear (assuming dry contact conditions) an experimental relationship between the volume of removed material and the frictional work [6,7] has been used. Particularly the relationship is able to directly evaluate the specific volume of removed material $\delta_{P_{w i}^{j k}(t)}(x, y)$ and $\delta_{P_{r i}^{j k}(t)}(x, y)$ related to the $i$ th contact points $P_{w i}^{j k}(t)$ and $P_{r i}^{j k}(t)$ on the $j$ th wheel and rail pair during the $k$ th of the $N_{c}$ dynamic simulations.

The calculation of $\delta_{P_{i}^{j k}(t)}(x, y)$ requires first of all the evaluation of the friction power developed by the tangential contact stresses; to this purpose the wear index $I_{W}$ (expressed in $\mathrm{N} / \mathrm{mm}^{2}$ ) is defined as follows:

$I_{W}=\frac{\mathbf{p}_{t} \cdot \mathbf{s}}{V}$.

This index, by means of appropriate experimental tests, can be correlated with the wear rate $K_{W}$ (expressed in $\mu \mathrm{g} /\left(\mathrm{m} \mathrm{mm}^{2}\right)$ ) which represents the mass of removed material for unit of distance traveled by the vehicle (expressed in $\mathrm{m}$ ) and for unit of surface (expressed in $\mathrm{mm}^{2}$ ). Wear tests carried out in the case of metal-metal contact with dry surfaces using a twin disc test machine can be found in literature [6]. The experimental 


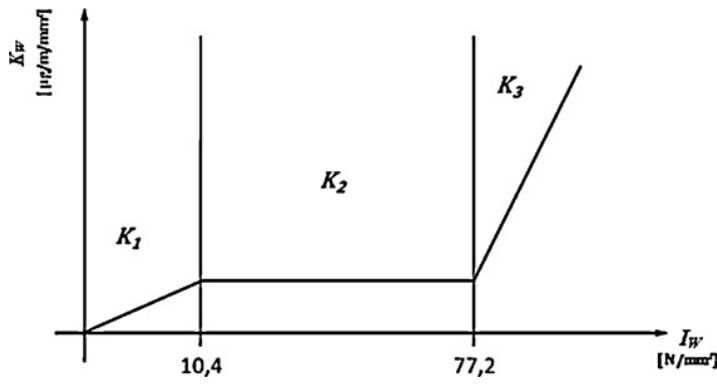

Fig. 12. Trend of the wear rate $K_{W}$.

relationship between $K_{W}$ and $I_{W}$ adopted for the wear model described in this work is the following (see Fig. 12):

$K_{W}\left(I_{W}\right)= \begin{cases}5.3 * I_{W} & I_{W}<10.4 \\ 55.0 & 10.4 \leq I_{W} \leq 77.2 \\ 61.9 * I_{W} & I_{W}>77.2\end{cases}$

Once the wear rate $K_{W}\left(I_{W}\right)$ is known (the same both for the wheel and for the rail), the specific volume of removed material on the wheel and on the rail (for unit of distance traveled by the vehicle and for unit of surface) can be calculated as follows (expressed in $\left.\mathrm{mm}^{3} /\left(\mathrm{m} \mathrm{mm}^{2}\right)\right)$ :

$\delta_{P_{w i}^{j k}(t)}(x, y)=K_{W}\left(I_{W}\right) \frac{1}{\rho}$

$\delta_{P_{r i}^{j k}(t)}(x, y)=K_{W}\left(I_{W}\right) \frac{1}{\rho}$

where $\rho$ is the material density (expressed in $\mathrm{kg} / \mathrm{m}^{3}$ ).

\subsection{Profile update}

The profile update strategy is the set of numerical procedures that allows the calculation of the new profiles of wheel $w_{n}\left(y_{w}\right)$ and rail $r_{n}\left(y_{r}\right)$ (the profiles at the next step), starting from the old profiles of wheel $w_{o}\left(y_{w}\right)$ and rail $r_{o}\left(y_{r}\right)$ (i.e. the profiles at the current step) and all the distributions of removed material $\delta_{p_{w i}^{j k}}(t)$ and $\delta_{P_{r i}^{j k}(t)}(x, y)$. The update strategy, besides evaluating the new profiles, is necessary for two additional reasons:

1. the necessity to remove the numerical noise that characterizes the distributions $\delta_{P_{i}^{j k}(t)}(x, y)$ and that, due to non physical alterations of the new profiles, can cause problems to the global contact model;

2. the need to mediate the distributions $\delta_{P_{i}^{j k}(t)}(x, y)$ in order to obtain a single profile both for the wheel and the rail as output of the wear model (as required by the specifications of Trenitalia and RFI).

The following main steps can be distinguished:

- Longitudinal integration:

$$
\begin{aligned}
& \frac{1}{2 \pi w\left(y_{w i}^{j k}\right)} \int_{-a(y)}^{+a(y)} \delta_{P_{w i}^{j k}(t)}(x, y) d x=\delta_{P_{w i}^{j k}(t)}^{t o t}(y) \\
& \frac{1}{l_{\text {track }}} \int_{-a(y)}^{+a(y)} \delta_{P_{r i}^{j k}(t)}(x, y) d x=\delta_{P_{r i}^{j k}(t)}^{t o t}(y)
\end{aligned}
$$

where $w\left(y_{w i}^{j k}\right)$ is the wheel radius evaluated in $y_{w i}^{j k}$ and $l_{\text {track }}$ is the length of the simulated track. This first integration sums, in the longitudinal direction, all the wear contributes inside the contact path and averages this quantity over the whole longitudinal

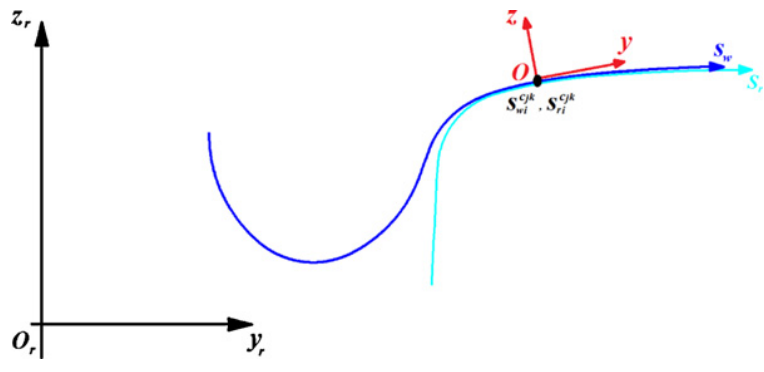

Fig. 13. Normal abscissa for the wheel and rail profile.

development of the wheel and of the rail (by means of the factors $1 / 2 \pi w\left(y_{w i}^{j k}\right)$ and $\left.1 / l_{\text {track }}\right)$; in other words it provides the mean value of removed material (expressed in $\mathrm{mm}^{3} /\left(\mathrm{m} \mathrm{mm}^{2}\right)$ ). The difference between the terms $1 / l_{\text {track }}$ and $1 / 2 \pi w\left(y_{w i}^{j k}\right)$ (the track length is much greater than the wheel circumference length) is the main cause that leads the wheel to wear much faster than the rail and consequently to a different scale of magnitude of the two investigated phenomena. This reflects the physical phenomena that the life of the rail is much greater than that of the wheel.

For this reason, as will be better explained in the following, it is necessary to develop a different strategy for the update of the wheel and rail profile respectively. In this research the following strategies have been adopted:

1. for the wheel update the mileage traveled by vehicle is considered. The total mileage $\mathrm{km}_{\text {tot }}$ (derived from the experimental data provided by Trenitalia and RFI) is subdivided into constant steps of length equal to $\mathrm{km}_{\text {step }}$;

2 . for the rail update, the total tonnage burden on the track [18] is considered. The vehicle number $N_{\text {tot }}$ corresponding to the total considered tonnage is subdivided into constant steps equal to $N_{\text {step. }}$.

- Track integration:

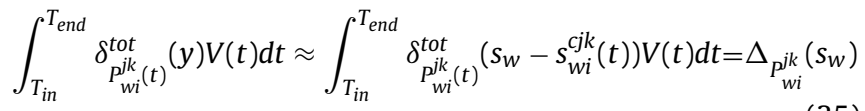

$\int_{T_{i n}}^{T_{\text {end }}} \delta_{P_{r i}^{j k}(t)}^{t o t}(y) V(t) d t \approx \int_{T_{i n}}^{T_{\text {end }}} \delta_{P_{r i}^{j k}(t)}^{t o t}\left(s_{r}-s_{r i}^{c j k}(t)\right) V(t) d t=\Delta_{P_{r i}^{j k}}\left(s_{r}\right)$

the track integration sums all the wear contributes coming from the dynamic simulation to obtain the depth of removed material for wheel $\Delta_{P_{w i}^{j k}}\left(s_{w}\right)$ and rail $\Delta_{P_{r i}^{j k}}\left(s_{r}\right)$ expressed in $\mathrm{mm}=\mathrm{mm}^{3} / \mathrm{mm}^{2}$. In order to have a better accuracy in the calculation of the worn profiles, the natural abscissas $s_{w}$ and $s_{r}$ of the curves $w\left(y_{w}\right)$ and $r\left(y_{r}\right)$ have been introduced. In particular the following relations locally hold (see Fig. 13):

$y \approx s_{w}-s_{w i}^{c j k}(t) \quad y \approx s_{r}-s_{r i}^{c j k}(t)$

$w\left(y_{w}\right)=w\left(y_{w}\left(s_{w}\right)\right)=\tilde{w}\left(s_{w}\right) \quad r\left(y_{r}\right)=r\left(y_{r}\left(s_{r}\right)\right)=\tilde{r}\left(s_{r}\right)$

where the natural abscissas of the contact points $s_{w i}^{c j k}$ and $s_{w i}^{c j k}$ can be evaluated from their positions $P_{w i}^{j k}$ and $P_{r i}^{j k}$.

- Sum on the contact points:

$$
\begin{aligned}
& \sum_{i=1}^{N_{P D C}} \Delta_{P_{w i}^{j k}}\left(s_{w}\right)=\Delta_{j k}^{w}\left(s_{w}\right) \\
& \sum_{i=1}^{N_{P D C}} \Delta_{P_{r i}^{j k}}\left(s_{r}\right)=\Delta_{j k}^{r}\left(s_{r}\right)
\end{aligned}
$$




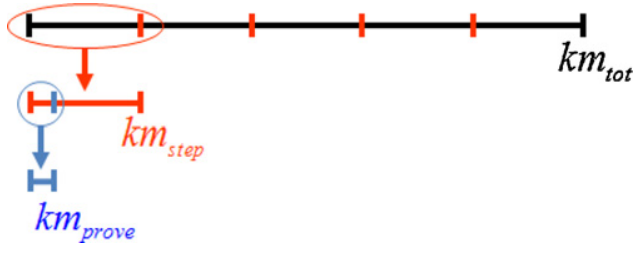

Fig. 14. Discretization of the total mileage.

where $N_{P D C}$ is the maximum number of contact points of each single wheel (and respectively of each single rail); since the number of contact points on the wheel-rail pair is usually less than $N_{P D C}$ and changes in the time during the dynamic simulation, it has been assumed that the wear contribution associated to the fictitious points is zero.

- Average on the vehicle wheels and on the dynamic simulations:

$$
\begin{aligned}
& \sum_{k=1}^{N_{c}} p_{k} \frac{1}{N_{w}} \sum_{j=1}^{N_{w}} \Delta_{j k}^{w}\left(s_{w}\right)=\bar{\Delta}^{w}\left(s_{w}\right) \\
& \sum_{k=1}^{N_{c}} p_{k} \frac{1}{N_{w}} \sum_{j=1}^{N_{w}} \Delta_{j k}^{r}\left(s_{r}\right)=\bar{\Delta}^{r}\left(s_{r}\right)
\end{aligned}
$$

where $N_{w}$ is the number of vehicle wheels while the $p_{k}, 1 \leq k \leq N_{c}$, $\sum_{k=1}^{N_{C}} p_{k}=1$ are the statistical weights associated to the various dynamic simulations derived from the statistical analysis. The average on the number of wheel-rail pairs has to be evaluated in order to obtain as output of the wear model a single average profile both for the wheel and for the rail (as required by Trenitalia and RFI).

- Scaling:

the aim of the scaling procedure is to amplify the small quantity of material removed during the $N_{c}$ dynamic simulations and, at the same time, to limit the computational load; using the almost linearity of the wear model with the traveled distance, it is possible to amplify the removed material by means of a scaling factor which increases the distance traveled by the vehicle.

The almost linearity of the wear model inside the discrete steps $k m_{\text {step }}$ in which the total mileage traveled $k m_{\text {tot }}$ is subdivided is a working hypothesis coming from the discrete approach of the model. It is based on the idea that the wear rate inside the simulated distance $\left(\mathrm{km}_{\text {prove }}\right)$ remains the same also inside the discrete step $\mathrm{km}_{\text {step }}$, since the considered vehicle always covers the same tracks of the statistical analysis both during the simulated distance $\left(k m_{\text {prove }}\right)$ and during the discrete step $\left(k m_{s t e p}\right)$.

In this work a constant discrete step has been chosen to update the wheel and rail profiles (see Fig. 14): in fact this method well adapts to the almost linear characteristic of the wear evolution. Furthermore it requires limited computational load without losing accuracy if compared with different suitable strategies as the adaptive step [7].

The evaluation of the discrete step, with the consequent scaling of $\bar{\Delta}^{w}\left(s_{w}\right)$ and $\bar{\Delta}^{r}\left(s_{r}\right)$, represents the major difference between wheel update and rail update:

1. the removed material on the wheel due to wear is proportional to the distance traveled by the vehicle; in fact a point of the wheel is frequently in contact with the rail in a number of times proportional to the distance. The following nomenclature can been introduced (see Fig. 14):

- $k m_{\text {tot }}$ is the total mileage traveled by the considered vehicle $\left(k m_{\text {tot }}\right.$ can be chosen depending on the purpose of the simulations, for example equal to the re-profiling intervals);

- $k m_{\text {step }}$ is the length of the discrete step in which the total mileage $k m_{\text {tot }}$ is subdivided;
- $k m_{\text {prove }}=l_{\text {track }}$ is the overall mileage traveled by the vehicle during the $N_{c}$ dynamic simulations; the necessity of acceptable computational time for the multibody simulations leads to adopt small value of the $\mathrm{km}_{\text {prove }}$ length and for this reason the relative removed material has to be scaled with a multiplicative factor.

Finally the material removed on the wheel has to be scaled according to the following law:

$\bar{\Delta}^{w}\left(s_{w}\right) \frac{k m_{\text {step }}}{k m_{\text {prove }}}=\bar{\Delta}^{w s c}\left(s_{w}\right)$.

The choice of the spatial step must be a good compromise between numerical efficiency and the accuracy required by the wear model. A $\mathrm{km}_{\text {step }}$ too small compared to $\mathrm{km}_{\text {tot }}$ would provide accurate results but excessive calculation times; the contrary happens with $\mathrm{km}_{\text {step }}$ too big compared to $\mathrm{km}_{\text {tot }}$.

2. the depth of rail wear is not proportional to the distance traveled by the vehicle; in fact the rail tends to wear out only in the zone where it is crossed by the vehicle and, increasing the traveled distance, the depth of removed material remains the same. On the other hand the rail wear is proportional to the total tonnage $M_{t o t}$ burden on the rail and thus to the total vehicle number $N_{\text {tot }}$ moving on the track. Therefore, if $N_{\text {step }}$ is the vehicle number moving in a discrete step, the quantity of rail removed material at each step will be:

$\bar{\Delta}^{r}\left(s_{r}\right) * N_{\text {step }}=\bar{\Delta}^{r s c}\left(s_{r}\right)$

where $N_{\text {step }}$ is calculated subdividing in constant step the vehicle number $N_{\text {tot }}$ corresponding to the total tonnage that has to be simulated; $N_{\text {tot }}$ can be obtained starting from the vehicle mass $M_{v}$ :

$N_{t o t}=\frac{M_{t o t}}{M_{v}}$.

- Smoothing of the removed material:

$\mathfrak{I}\left[\bar{\Delta}^{w s c}\left(s_{w}\right)\right]=\bar{\Delta}_{s m}^{w s c}\left(s_{w}\right)$

$\Im\left[\bar{\Delta}^{r s c}\left(s_{r}\right)\right]=\bar{\Delta}_{s m}^{r s c}\left(s_{r}\right)$;

the smoothing of the removed material function is necessary to remove the numerical noise that affects this quantity and that would be passed to the new profiles $\tilde{w}_{n}\left(s_{w}\right)$ and $\tilde{r}_{n}\left(s_{r}\right)$ of wheel and rail causing problems to the global contact model. To this end, a discrete filter (i.e. a moving average filter with window size equal to $1 \% \div 5 \%$ of the total number of points in which the profiles are discretized) has been used; obviously the discrete filter has to conserve the mass.

- Profile update:

$$
\begin{aligned}
& \left(\begin{array}{c}
y_{w}\left(s_{w}\right) \\
\tilde{w}_{o}\left(s_{w}\right)
\end{array}\right)-\bar{\Delta}_{s m}^{w^{s c}}\left(s_{w}\right) \mathbf{n}_{w}^{r} \quad \stackrel{\text { re-parameterization }}{\longrightarrow}\left(\begin{array}{c}
y_{w}\left(s_{w}\right) \\
\tilde{w}_{n}\left(s_{w}\right)
\end{array}\right) \\
& \left(\begin{array}{c}
y_{r}\left(s_{r}\right) \\
\tilde{r}_{o}\left(s_{r}\right)
\end{array}\right)-\bar{\Delta}_{s m}^{r s c}\left(s_{r}\right) \mathbf{n}_{r}^{r} \quad \stackrel{\text { re-parameterization }}{\longrightarrow}\left(\begin{array}{c}
y_{r}\left(s_{r}\right) \\
\tilde{r}_{n}\left(s_{r}\right)
\end{array}\right) \text {; }
\end{aligned}
$$

the last step consists in the update of the old profiles $\tilde{w}_{o}(s)=$ $w_{o}(y)$ and $\tilde{r}_{o}\left(s_{r}\right)=r_{o}\left(y_{r}\right)$ to obtain the new profiles $\tilde{w}_{n}(s)=w_{n}(y)$ and $\tilde{r}_{n}\left(s_{r}\right)=r_{n}\left(y_{r}\right)$; since the removal of material occurs in the normal direction to the profiles $\left(\mathrm{n}_{\mathrm{w}}^{\mathrm{r}}\right.$ and $\mathrm{n}_{\mathrm{r}}^{\mathrm{r}}$ are the outgoing unit vector for the wheel and rail profile respectively), once removed the quantities $\bar{\Delta}_{s m}^{w s c}\left(s_{w}\right)$ and $\bar{\Delta}_{s m}^{r s c}\left(s_{r}\right)$, a re-parameterization of the profiles is needed in order to obtain again curves parameterized by means of the curvilinear abscissa. 


\section{Wear model validation}

In this section the wear model validation phase will be presented. Initially, the set of $N_{c}$ curvilinear tracks, on which the dynamic simulations of the DMU Aln 501 Minuetto vehicle have been performed, will be introduced (tracks extracted starting from the statistical analysis of the Aosta-Pre Saint Didier track, the data of which has been provided by RFI); moreover the wear control parameters for the wheel and rail will be defined (the flange height FH, the flange thickness FT, the flange steepness QR and the quota QM for the rail). Then the experimental data (provided by Trenitalia) measured on the Aosta-Pre Saint Didier track and their processing will be introduced. Finally, the simulation strategy used to analyze the wear both on the wheel and on the rail will be described and the results obtained with the wear model will be analyzed and compared with the experimental data.

\subsection{Statistical analysis of the Aosta-Pre Saint Didier track}

Starting from the data of the whole Aosta-Pre Saint Didier track (provided by RFI), the statistical analysis has been performed by dividing the line both in radius classes (determined by $R_{\min }$ and $R_{\max }$ ) and in superelevation classes (determined by $h_{\min }$ and $h_{\max }$ ) [11]. More particularly five superelevation subclasses are defined for each radius class. The subclasses that do not include curve have not been taken into account in the definition of the set of $N_{c}$ tracks. All the $N_{c}$ curved tracks are shown in Table 3.

The set consists in $N_{c}=18$ distinct elements (17 real curves and the straight line) characterized by the radius value $R_{c}$, the superelevation value $H$, the traveling speed $V$ and the statistical weight $p_{k}$ (with $1 \leq k \leq N_{c}$ ) that represents the frequency with which each curve appears on the considered railway track (Aosta-Pre Saint Didier line). The radii $R_{c}$ are calculated by means of the weighted mean on all the curve radii included in the corresponding superelevation subclass (the weighted factor is the length of the curves in the real track). For each subclass, the value $H$ is the most frequent superelevation value among the standard values that characterize the curves of the considered superelevation subclass. The traveling speeds $V$ are calculated imposing a threshold value on the uncompensated acceleration $a_{n c}^{\lim }=0.6 \mathrm{~m} / \mathrm{s}^{2}$ :

$\frac{\tilde{V}^{2}}{R_{c}}-\frac{H}{s} g=a_{n c}^{l i m}$

where $s$ is the railway gauge and $g$ is the gravity acceleration. The estimated speed $\tilde{V}$ has been then compared with the maximum velocity $V_{\max }$ on the line to get the desired traveling speed $V=$ $\min \left(\tilde{V}, V_{\max }\right)$

\subsection{Wear control parameters}

The reference quotas FH, FT and QR are introduced in order to estimate the wheel profile evolution due to the wear without necessarily knowing the whole profile shape (see Fig. 15). According to these quotas the user will be able both to established when the worn wheel profile will have to be re-profiled and to detect if the wear compromises the dynamical stability of the vehicle [19].

The procedure to define the reference quotas is the following:

1. first of all the point $P 0$ is defined on the profile, at $70 \mathrm{~mm}$ from the internal vertical face of the wheel;

2. then the point $P 1$ is introduced on the profile, $2 \mathrm{~mm}$ under the flange vertex;

3. finally the point $P 2$ is determined on the profile, $10 \mathrm{~mm}$ under the point $P O$;

4. the wear control parameters are then calculated as follows: the flange thickness FT is the horizontal distance between the

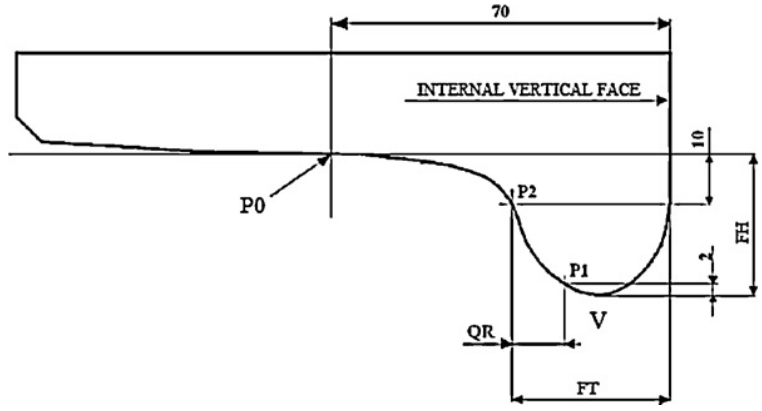

Fig. 15. Definition of the wheel wear control parameters.

internal vertical face and the point $P 2$; the flange steepness $Q R$ is the horizontal distance between the points $P 1$ and $P 2$, while the flange height $\mathrm{FH}$ is the vertical distance between $P 0$ and the flange vertex (all the distances are considered positive).

An additional control parameter is then introduced to evaluate the evolution of rail wear. Particularly the QM quota is defined as the rail head height in the point $y_{r}=760 \mathrm{~mm}$ with respect to the center line of the track: this $y_{r}$ value depends on the railway gauge (equal to $1435 \mathrm{~mm}$ in the Aosta-Pre Saint Didier line) and on the laying angle $\alpha_{p}$ of the track (equal to $1 / 20 \mathrm{rad}$ ). Physically the QM quota gives information on the rail head wear (see Fig. 16).

\subsection{Experimental data and their processing}

The experimental data provided by Trenitalia and RFI are related only to the wheel wear and consists in the wear control parameters measured as a function of the total distance traveled by the considered vehicle DMU Aln 501 Minuetto; particularly, the data have been measured on three different vehicles operating on the same track that are conventionally called DM061, DM068, DM082.

As can be seen by example in Table 4 for the vehicle MD061, the reference quota values have been measured for all the vehicle wheels (each vehicle has eight wheelsets as specified in Section 3.1). However the following data processing has been necessary in order to obtain a single wheel profile that could be effectively compared with the profile extracted from the numerical simulation and to reduce the measurement errors:

1. initially the arithmetic mean on all the sixteen vehicle wheels has been evaluated; the mean is necessary to obtain a single wheel profile and, at the same time, to reduce the measurement errors affecting the experimental data;

2. then a scaling of the quota values has been carried out in order to delete the offset on the initial value of the considered quantities: this procedure imposes that all the wear control parameters start from their nominal values (the standard values for the ORE S 1002 profile have been used) in order to remove the initial differences among the vehicles due to measurement errors;

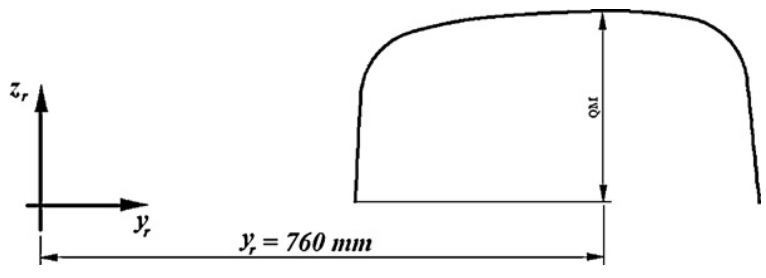

Fig. 16. Definition of rail wear control parameter. 
Table 3

Data of the curvilinear tracks of the statistical analysis.

\begin{tabular}{|c|c|c|c|c|c|c|}
\hline$R_{\min }(\mathrm{m})$ & $R_{\max }(\mathrm{m})$ & Superelevation $h_{\min }-h_{\max }(\mathrm{mm})$ & $R_{c}(\mathrm{~m})$ & $H(\mathrm{~mm})$ & $V(\mathrm{~km} / \mathrm{h})$ & $p_{k}(\%)$ \\
\hline 147.1 & 156.3 & $\begin{array}{c}0 \\
10-40 \\
60-80 \\
90-120 \\
130-160\end{array}$ & $\begin{array}{r}- \\
- \\
- \\
150 \\
-\end{array}$ & 120 & 55 & 0.77 \\
\hline 156.3 & 166.7 & $\begin{array}{c}0 \\
10-40 \\
60-80 \\
90-120 \\
130-160\end{array}$ & $\begin{array}{r}- \\
- \\
- \\
160 \\
165\end{array}$ & $\begin{array}{l}110 \\
140\end{array}$ & $\begin{array}{l}55 \\
55\end{array}$ & $\begin{array}{l}0.48 \\
0.56\end{array}$ \\
\hline 166.7 & 178.6 & $\begin{array}{c}0 \\
10-40 \\
60-80 \\
90-120 \\
130-160\end{array}$ & $\begin{array}{r}- \\
- \\
- \\
170 \\
175\end{array}$ & $\begin{array}{l}110 \\
130\end{array}$ & $\begin{array}{l}55 \\
55\end{array}$ & $\begin{array}{l}0.82 \\
1.55\end{array}$ \\
\hline 178.6 & 192.3 & $\begin{array}{c}0 \\
10-40 \\
60-80 \\
90-120 \\
130-160\end{array}$ & $\begin{array}{r}- \\
- \\
- \\
190 \\
180\end{array}$ & $\begin{array}{l}100 \\
130\end{array}$ & $\begin{array}{l}55 \\
55\end{array}$ & $\begin{array}{l}8.37 \\
0.45\end{array}$ \\
\hline 192.3 & 208.3 & $\begin{array}{c}0 \\
10-40 \\
60-80 \\
90-120 \\
130-160\end{array}$ & $\begin{array}{r}- \\
- \\
- \\
200 \\
200\end{array}$ & $\begin{array}{r}90 \\
130\end{array}$ & $\begin{array}{l}55 \\
60\end{array}$ & $\begin{array}{l}20.64 \\
4.00\end{array}$ \\
\hline 208.3 & 227.3 & $\begin{array}{c}0 \\
10-40 \\
60-80 \\
90-120 \\
130-160\end{array}$ & $\begin{array}{r}- \\
- \\
220 \\
220 \\
-\end{array}$ & $\begin{array}{r}80 \\
100\end{array}$ & $\begin{array}{l}55 \\
55\end{array}$ & $\begin{array}{l}0.70 \\
3.76\end{array}$ \\
\hline 227.3 & 250.0 & $\begin{array}{c}0 \\
10-40 \\
60-80 \\
90-120 \\
130-160\end{array}$ & $\begin{array}{r}- \\
- \\
240 \\
240 \\
-\end{array}$ & $\begin{array}{r}80 \\
110\end{array}$ & $\begin{array}{l}55 \\
60\end{array}$ & $\begin{array}{l}7.26 \\
5.28\end{array}$ \\
\hline 250.0 & 312.5 & $\begin{array}{c}0 \\
10-40 \\
60-80 \\
90-120 \\
130-160\end{array}$ & $\begin{array}{r}- \\
- \\
270 \\
270 \\
-\end{array}$ & $\begin{array}{l}70 \\
90\end{array}$ & $\begin{array}{l}55 \\
60\end{array}$ & $\begin{array}{l}3.91 \\
5.29\end{array}$ \\
\hline 312.5 & 416.7 & $\begin{array}{c}0 \\
10-40 \\
60-80 \\
90-120 \\
130-160\end{array}$ & $\begin{array}{r}- \\
- \\
370 \\
345 \\
-\end{array}$ & $\begin{array}{r}60 \\
100\end{array}$ & $\begin{array}{l}55 \\
70\end{array}$ & $\begin{array}{l}2.26 \\
1.63\end{array}$ \\
\hline 416.7 & $\infty$ & 0 & $\infty$ & 0 & 70 & 32.27 \\
\hline
\end{tabular}

3. the arithmetic mean on the three vehicle MD061, MD068, MD082 has not been carried out, in order to maintain a dispersion range for the experimental data.

The experimental data, properly processed, are summarized in Table 5 . As can be seen, the flange height $\mathrm{FH}$ remains approximately constant because of the low mileage traveled by the vehicles, while the flange thickness FT and the flange steepness QR decrease almost linearly and highlight, according to the characteristics of the track, the wear concentration in the wheel flange.

\subsection{Simulation strategy}

In this section the simulation campaign performed to study the wear on wheel and rail is described. As explained in Section 4.3, the two phenomena evolve according to different time scales (several orders of magnitude) and a fully simulation of such events would require a too heavy computational effort. For this reason the following specific algorithm has been adopted for updating the profiles:

1. both for the wheel and for the rail five discrete steps have been chosen, $n_{s w}=5$ and $n_{s r}=5$, so to have a good compromise between calculation times and result accuracy:

(a) the choice of the wheel $\mathrm{km}_{\text {step }}$ (see Section 4.3 ) has been made considering the whole distance traveled equal to $k m_{\text {tot }} \approx 3500 \mathrm{~km}$ (got from experimental data); thus the single step length will be:

$k m_{\text {step }}=\frac{k m_{\text {tot }}}{n_{\text {sw }}} \approx 700(\mathrm{~km})$.

(b) to estimate the vehicle number $N_{\text {tot }}$ (see Section 4.3) a criterion present in literature (based on the total tonnage burden on the track) has been used [18]. Particularly there is a proportionality relationship between tonnage and wear: a rail wear of $1 \mathrm{~mm}$ on the rail head height every $100 \mathrm{Mt}$ (millions of tons) of accumulated tonnage. In order to obtain an 


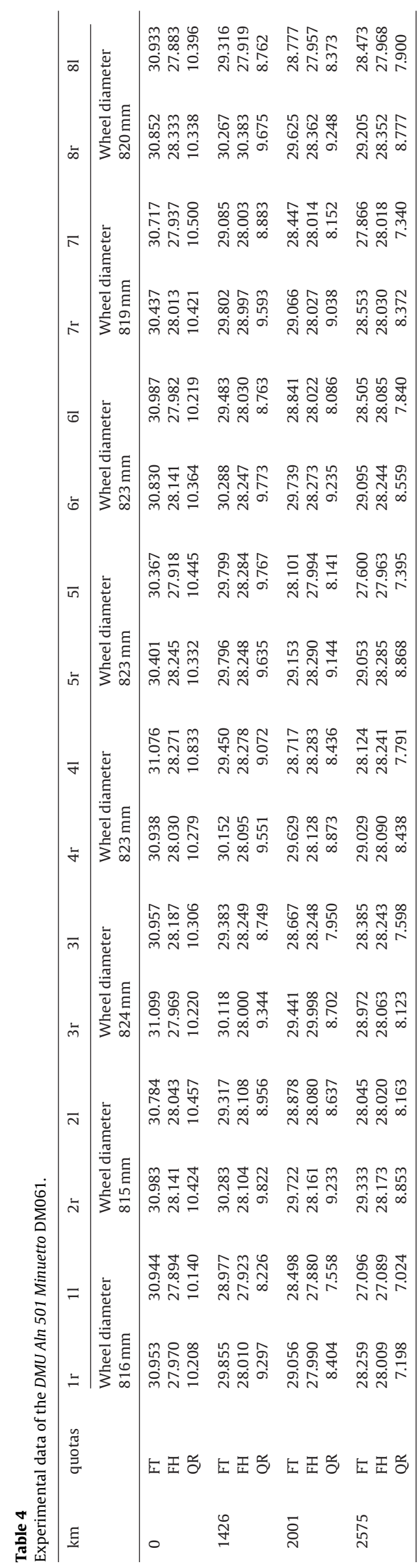

appreciable rail wear, a maximum value of removed material depth of $2 \mathrm{~mm}$ on the rail head height has been hypothesized (naturally this value can be changed according to the requirements of the simulation). The number of vehicles, of known mass $M_{v}$ (see Table 1 ), which should evolve on the track to reach the $200 \mathrm{Mt}$, has been therefore calculated:

$N_{\text {tot }}=\frac{M t o t}{M_{v}} \approx 2,000,000$

and then:

$N_{\text {step }}=\frac{N_{\text {tot }}}{n_{\text {sr }}} \approx 400,000$.

2. the wear evolution on wheel and rail has been decoupled because of the different scales of magnitude:

(a) while the wheel wear evolves, the rail is supposed to be constant: in fact, in the time scale considered, the rail wear variation is negligible.

(b) because of the time scale characteristic of the rail wear, each discrete rail profile comes in contact, with the same frequency, with each possible wheel profile. For this reason, for each rail profile, the whole wheel wear evolution (from the original profile to the final profile) has been simulated.

Based on the two previous hypotheses, the simulations have been carried out according to the following strategy:

Wheel profile evolution at first rail step : $w_{i}^{0}$

$p_{1,1}\left\{\left(\begin{array}{cc}w_{0}^{0} & r_{0} \\ & \end{array}\right) \rightarrow\left(\begin{array}{ll}w_{1}^{0} & r_{0} \\ & \end{array}\right) \rightarrow \cdots \rightarrow\left(\begin{array}{ll}w_{4}^{0} & r_{0} \\ & \end{array}\right) \rightarrow w_{5}^{0}\right.$ Average on the rails $r_{1}^{(i+1)}$ for the calculation of the second rail step : $r_{1}$

$p_{1,2}\left\{\left(\begin{array}{cc}w_{0}^{0} & r_{0} \\ w_{1}^{0} & r_{0} \\ \vdots & \vdots \\ w_{4}^{0} & r_{0}\end{array}\right) \rightarrow\left(\begin{array}{c}r_{1}^{(1)} \\ r_{1}^{(2)} \\ \vdots \\ r_{1}^{(5)}\end{array}\right) \rightarrow r_{1}\right.$

Wheel profile evolution at fourth rail step : $w_{i}^{4}$

$p_{5,1}\left\{\left(\begin{array}{ll}w_{0}^{4} & r_{4} \\ & \end{array}\right) \rightarrow\left(\begin{array}{ll}w_{1}^{4} & r_{4} \\ & \end{array}\right) \rightarrow \cdots \rightarrow\left(\begin{array}{ll}w_{4}^{4} & r_{4} \\ & \end{array}\right) \rightarrow w_{5}^{4}\right.$ Average on the rails $r_{5}^{(i+1)}$ for the calculation of the fifth rail step : $r_{5}$

$p_{5,2}\left\{\left(\begin{array}{cc}w_{0}^{4} & r_{4} \\ w_{1}^{4} & r_{4} \\ \vdots & \vdots \\ w_{4}^{4} & r_{4}\end{array}\right) \rightarrow\left(\begin{array}{c}r_{5}^{(1)} \\ r_{5}^{(2)} \\ \vdots \\ r_{5}^{(5)}\end{array}\right) \rightarrow r_{5}\right.$

where $w_{i}^{j}$ indicates the $i$ th step of the wheel profile that evolves on $j$ th step of the rail profile $r_{j}$. The initial profiles $w_{0}^{j}$ are always 
Table 5

Experimental data processed.

\begin{tabular}{lclll}
\hline Vehicle & Distance traveled $(\mathrm{km})$ & $\mathrm{FH}(\mathrm{mm})$ & $\mathrm{FT}(\mathrm{mm})$ & $\mathrm{QR}(\mathrm{mm})$ \\
\hline DM061 & 0 & 28.0 & 32.5 & 10.8 \\
& 1426 & 28.2 & 31.5 & 9.8 \\
& 2001 & 28.1 & 30.8 & 9.1 \\
& 2575 & 28.0 & 30.2 & 8.6 \\
DM068 & 0 & 28.0 & 32.5 & 10.8 \\
& 1050 & 28.0 & 31.8 & 10.0 \\
& 2253 & 28.0 & 30.2 & 8.5 \\
& 2576 & 28.0 & 30.0 & 8.4 \\
DM082 & 0 & 28.0 & 32.5 & 10.8 \\
& 852 & 28.0 & 32.3 & 10.6 \\
& 1800 & 28.0 & 31.3 & 9.6 \\
& 2802 & 28.0 & 30.3 & 8.7 \\
& 3537 & 27.6 & 30.0 & 8.3 \\
\hline
\end{tabular}

the same for each $j$ and correspond to the unworn wheel profile (ORE S 1002).

Initially the wheel (starting from the unworn profile $w_{0}^{0}$ ) evolves on the unworn rail profile $r_{0}$ in order to produce the discrete wheel profiles $w_{0}^{0}, w_{1}^{0}, \ldots, w_{5}^{0}$ (step $p_{1,1}$ ). Then the virtual rail profiles $r_{1}^{(i+1)}$, obtained by means of the simulations $\left(w_{i}^{0}, r_{0}\right)$ with $0 \leq i \leq 4$, are arithmetically averaged so as to get the update rail profile $r_{1}\left(\operatorname{step} p_{1,2}\right)$. This procedure can be repeated $n_{s r}$ times in order to perform all the rail discrete steps (up to the step $p_{5,2}$ ).

The computational effort required by the simulation strategy is the following:

(a) in the wheel wear study, for each update of the rail profile $r_{j}$, the whole wheel wear loop $w_{i}^{j}$ with $0 \leq i \leq 4$ ( $n_{s w}$ steps of simulation) is simulated. The computational effort results of $n_{s w} \times n_{s r}=25$ steps both for the dynamic analysis (in Simpack Rail) and for the wear model necessary to calculate the removed material on the wheel (in Matlab). So the total number of simulation steps are $2\left(n_{s w} \times n_{s r}\right)=50$.

(b) in the rail wear study the dynamic analyses are the same as the previous case because for each rail step the wheel profiles $w_{i}^{j}(0 \leq i \leq 4)$ are simulated on $r_{j}$ in order to obtain $r_{j}^{(i+1)}$ and thus the updated rail profile $r_{j+1}$ by means of an arithmetic mean. Therefore, no additional dynamical analyses are needed. In this case only the wear model steps must be simulated so as to get the removed material on the rail. Consequently the total number of simulation steps is $n_{s w} \times n_{s r}=25$.

The characteristics of the processor used in the simulations and the mean computational times relative to each discrete step of the whole model loop (dynamical simulation and wear simulation) are briefly summarized in Table 6 .

\subsection{Evolution of wear control parameters}

In this section the evolution of the wheel reference quotas numerically evaluated by means of the wear model (flange thickness FT, flange height FH and flange steepness QR) will be compared with the experimental data concerning the three DMUs Aln 501

Table 6

Computational time.

\begin{tabular}{lll}
\hline Processor & \multicolumn{2}{c}{ Computational time } \\
\cline { 2 - 3 } & $\begin{array}{l}\text { Dynamic simulation } \\
\text { (Simpack Rail) }\end{array}$ & $\begin{array}{l}\text { Wear simulation } \\
\text { (Matlab) }\end{array}$ \\
\hline $\begin{array}{l}\text { INTEL Xeon CPU E 5430 } \\
\text { 2.66 GHz 8 GB RAM }\end{array}$ & $2 \mathrm{~h} \mathrm{2 \textrm {s }}$ & $31 \mathrm{~s}$ \\
\hline
\end{tabular}

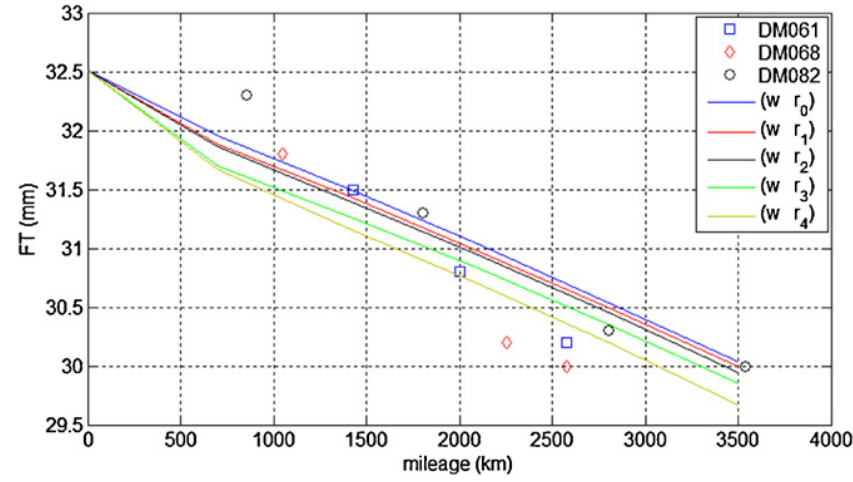

Fig. 17. FT dimension progress.

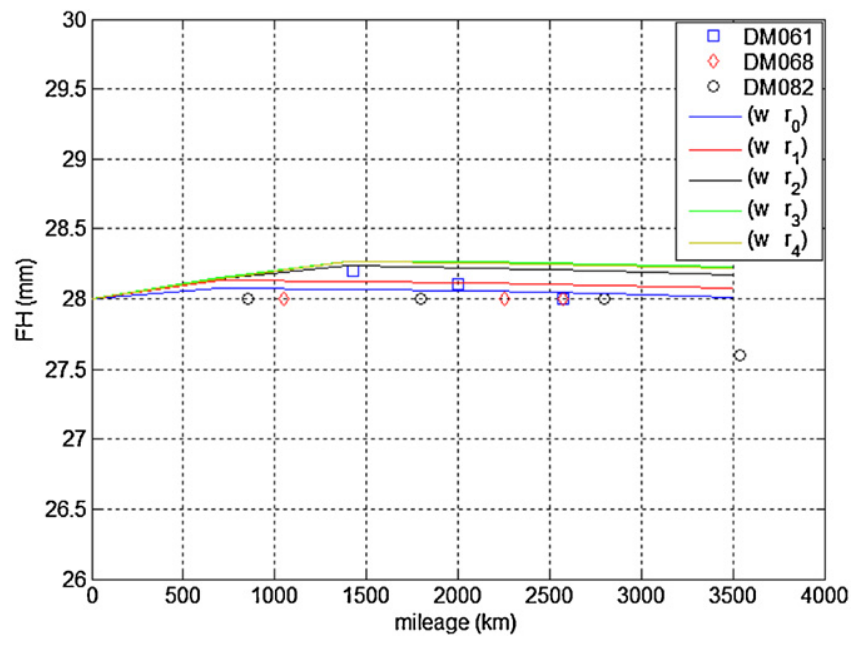

Fig. 18. FH dimension progress.

Minuetto vehicles. Furthermore the rail reference quota QM evolution will be shown and compared with the criterion present in literature based on the total tonnage burden on the track [18].

The progress of FT dimension, for the $n_{s r}$ discrete step of the rail, is shown in Fig. 17 as a function of the mileage; as it can be seen, the decrease of the dimension is almost linear with the traveled distance except in the first phases, where the profiles are still not conformal enough. The FH quota progress is represented in Fig. 18 and shows that, due to the presence of many sharp curves in the statistical analysis of the track and to the few kilometers traveled, the wheel wear is mainly localized on the flange rather than on the tread and the flange height remains near constant in agreement with experimental data. The QR trend is shown in Fig. 19: also the flange steepness decreases almost linearly, leading to an increase of

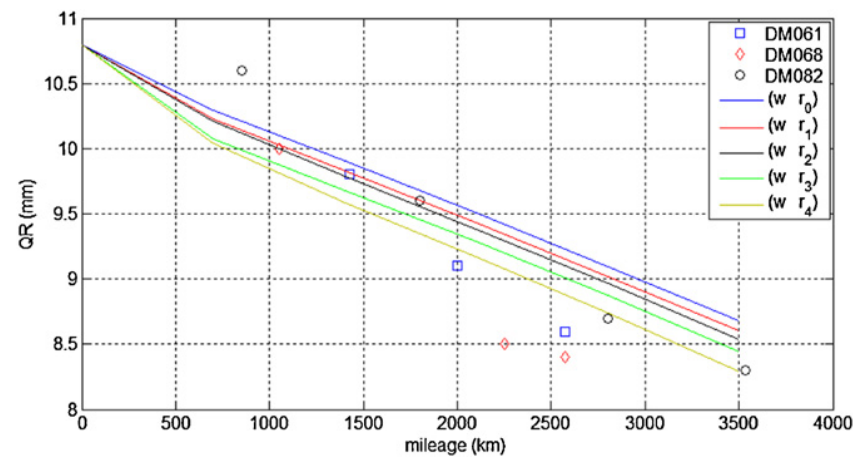

Fig. 19. QR dimension progress. 


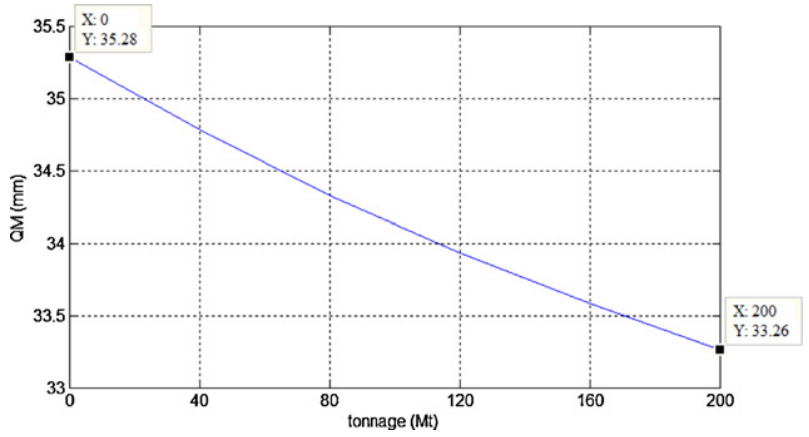

Fig. 20. QM dimension progress.

the conicity of the flange. Finally, the evolution of the wheel control parameters remains quantitatively and qualitatively similar as the rail wear raises.

Although the simulated mileage is quite short considering the mean traveled distance between two turnings of the wheels in a standard scenery (in fact the FH quota remains almost constant), the variations of the FT and QR dimensions are remarkable and it highlights the wear problems affecting the vehicle DMU Aln 501 Minuetto running along the railway line taken into account.

In conclusion, the comparisons show that the outputs of the wear model are consistent with the experimental data, both for the flange dimension (FH, FT) and for the conicity (QR); the slightly steeper development of the experimental data than the simulation can be explained with the dispersion of the experimental data and with wear mechanisms, like plastic and pitting wear, not considered in the developed wear model.

Finally, the QM evolution for the analysis of the rail wear is presented in Fig. 20 and shows the almost linear dependence between the rail wear and the total tonnage burden on the track: the amount of removed material on the rail profile is in agreement with the criterion present in literature $(1 \mathrm{~mm}$ on the rail head height every $100 \mathrm{Mt}$ of accumulated tonnage).

\subsection{Evolution of the wheel and rail profiles}

The wear evolution on the wheel profiles evolving on each rail $r_{j}$ (with $0 \leq j \leq n_{s r}$ and $n_{s r}=5$ ) is presented in the following (see Figs. 21 and 23-27). As stated previously, the wheel profile evolution is described by means of $n_{s w}=5$ steps and the spatial step $k m_{s t e p}$ has been chosen equal to $700 \mathrm{~km}$, since the total mileage $\mathrm{km}_{\text {tot }}$ is $3500 \mathrm{~km}$. Fig. 22 shows the cumulative distributions of removed material in vertical direction $z_{w}$ on the wheel profile at first rail step $\sigma_{w_{K w}}\left(y_{w}\right)=\sum_{i=1}^{K w} \sigma_{i}^{w}\left(y_{w}\right)$ as a function of $y_{w}\left(1 \leq K w \leq n_{s w}\right)$, where $\sigma_{i}^{w}\left(y_{w}\right)$ is the removed material between two subsequent discrete steps of the wheel profile evolution.

The quite limited distance traveled by the vehicle justifies the low wear on the wheel tread and entails a small reduction of the rolling radius. However the high tortuosity of the considered track leads to appreciable wear on the wheel flange. In Figs. 21 and 23-27, focusing on the flange zone, the higher wear rate during the first

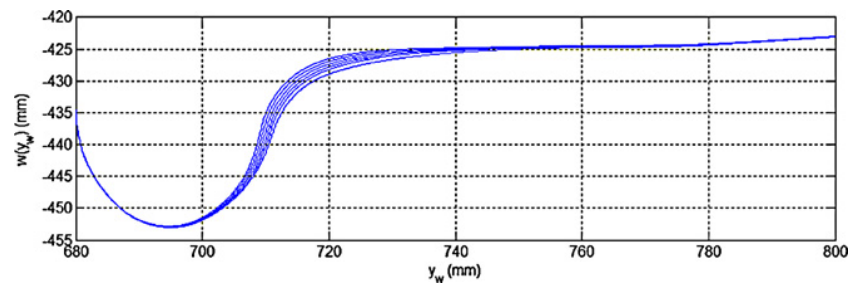

Fig. 21. Evolution of the wheel profile on the $r_{0}$ rail.

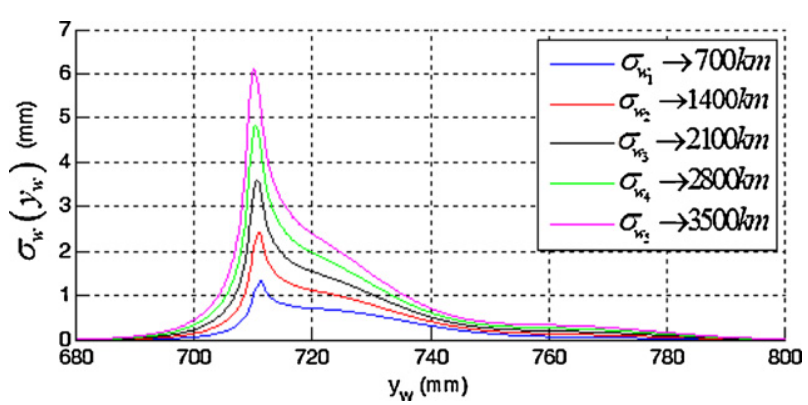

Fig. 22. Cumulative distributions of the removed wheel material.

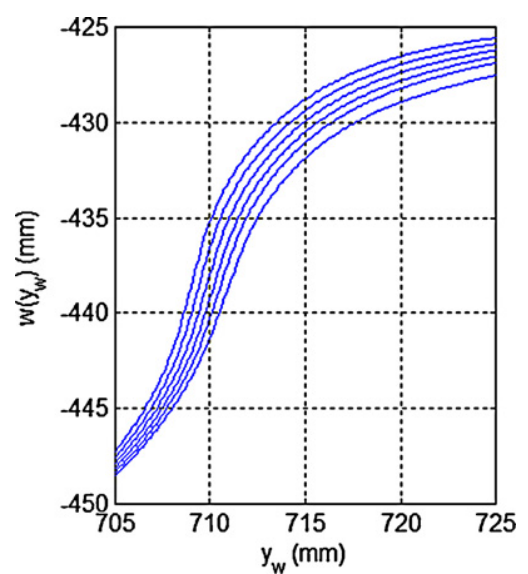

Fig. 23. Evolution of the wheel profile on the $r_{0}$ rail in the flange zone.

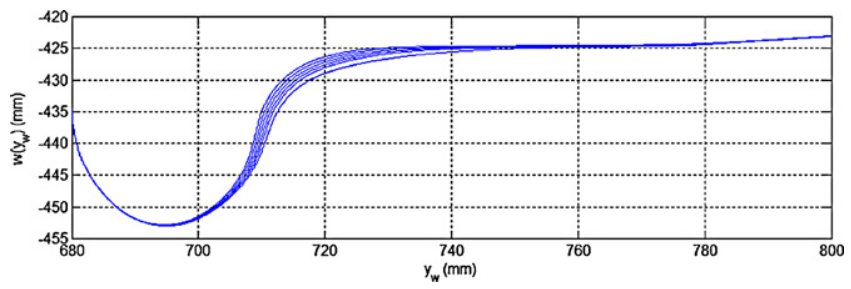

Fig. 24. Evolution of the wheel profile on the $r_{1}$ rail.

steps can be observed because of the initial non-conformal contact that characterizes the coupling between the ORE S 1002 wheel profile and the UIC 60 rail profile with an inclination of $\alpha_{p}=1 / 20 \mathrm{rad}$; then the rate decreases becoming more regular and constant in the last steps, when the contact is more and more conformal.

Also as regards the wheel profile evolution (as for the reference quotas) the trend remains quantitatively and qualitatively the same as the rail wear raises (see Figs. 24-27).

In Figs. 28 and 30 the evolution of the rail profile is shown, described by means of $n_{s r}=5$ discrete step and with $N_{\text {step }}$ equal to 400,000 (the vehicle number $N_{\text {tot }}$, corresponding to the total studied tonnage $M_{t o t}$, is 2,000,000). In Fig. 29 the cumulative distribution of the removed material on the rail proflie in $z_{r}$

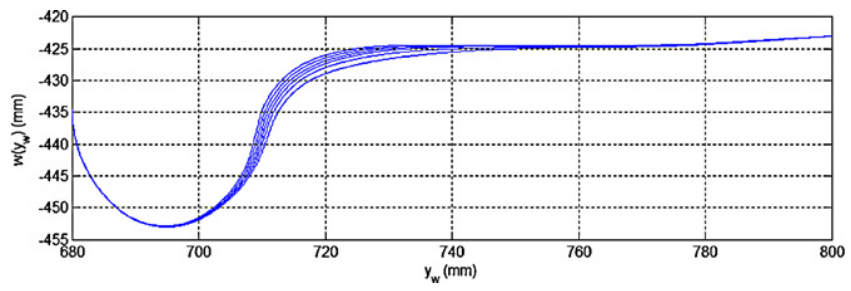

Fig. 25. Evolution of the wheel profile on the $r_{2}$ rail. 


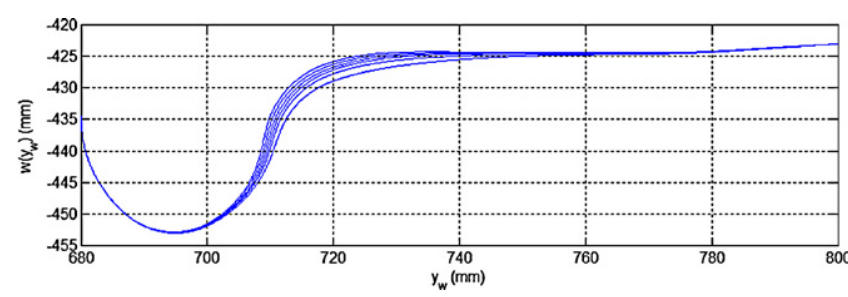

Fig. 26. Evolution of the wheel profile on the $r_{3}$ rail.

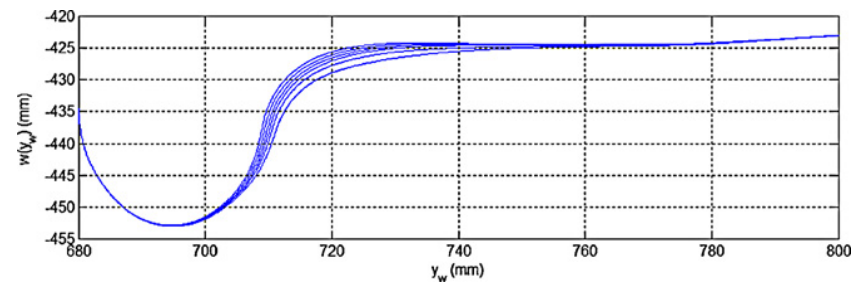

Fig. 27. Evolution of the wheel profile on the $r_{4}$ rail.

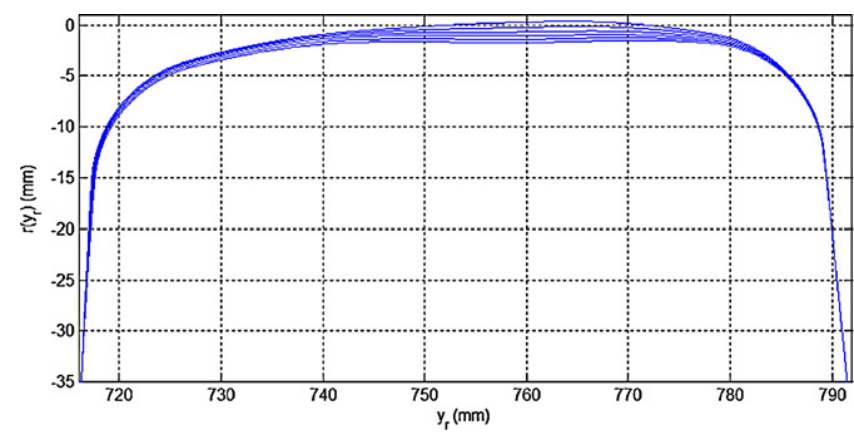

Fig. 28. Evolution of the rail profile.

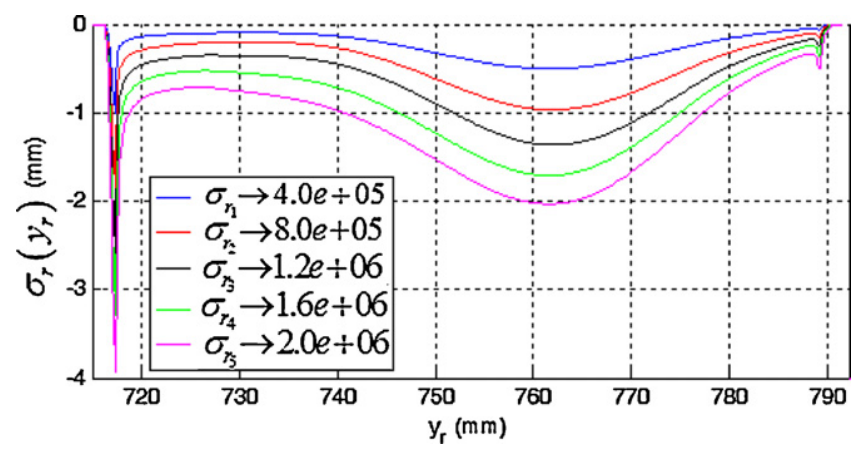

Fig. 29. Cumulative distributions of the removed rail material.

direction $\sigma_{r_{K r}}\left(y_{r}\right)=\sum_{i=1}^{K r} \sigma_{i}^{r}\left(y_{r}\right)$ expressed as a function of $y_{r}$ (with $\left.1 \leq K r \leq n_{s r}\right)$ is shown, where $\sigma_{i}^{r}\left(y_{r}\right)$ is the removed material between two subsequent discrete steps of the rail profile evolution. The value of total tonnage taken into account $\left(M_{t o t}=200 \mathrm{Mt}\right)$ causes an appreciable wear on the rail head, while it is not sufficient to produce an high wear also on the rail shouder (Fig. 30).

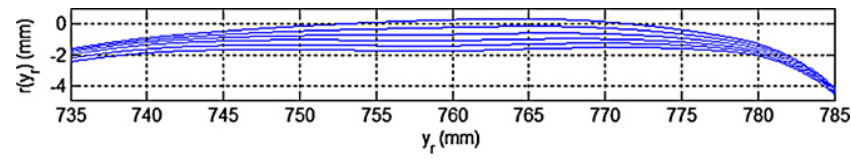

Fig. 30. Evolution of the rail profile in head zone.

\section{Conclusions}

In this work the Authors presented a complete model for the wheel and rail wear prediction in railway application, developed thanks to the collaboration with Trenitalia S.p.A and Rete Ferroviaria Italiana (RFI), which provided the necessary technical and experimental data for the model validation. The whole model is made up of two mutually interactive parts. The first one evaluates the vehicle dynamics and comprises both the multibody model of the vehicle implemented in Simpack Rail and a global wheel-rail contact model (developed by the Authors in previous works) for the calculation of the contact points and of the contact forces. The second one is the wear model which, starting from the outputs of the multibody simulations, evaluates the amount of material to be removed due to wear. The interaction between the two parts is not a continuous time process but occurs at discrete steps; consequently the evolution of the wheel and rail geometry is described through several intermediate profiles.

In particular a suitable update algorithm has been developed in order to consider the different time scale characterizing the wheel and rail wear evolution: the wheel wear has been studied basing on the distance traveled by vehicle, while the rail wear has been evaluated basing on the total tonnage burden on the railway track.

The whole model has been validated on a critical scenario in terms of wear in Italian railways: the ALSTOM DMU Aln 501 Minuetto circulating on the Aosta-Pre Saint Didier railway line. A statistical approach to describe the track has been used to reduce the total computational effort.

As regards the wheel wear, the results obtained from the dynamic simulations have been compared with the experimental data provided by Trenitalia while, concerning the rail wear, the comparison with experimental criteria based on the total tonnage burden on the railway track have been considered. The developed model reproduces quite good the evolution of all the profile characteristic dimensions describing in satisfying way the wear progress both on the wheel and on the rail.

The results obtained for the wheel profile evolution highlights how, in the particular operating conditions of the studied railway line, the wear is quite severe and strongly localized on the wheel flange, leading to frequent maintenance interventions. As regards the rail profile evolution, the wear is mainly focused on the rail head due to the initial low conformity of the coupling ORE S 1002UIC 60 with $\alpha_{p}=1 / 20$ rad while the total tonnage considered is not sufficient to produce an high wear also on the shouder of the rail.

Future developments will be based on further experimental data (relative to other railway track with a higher mileage than the Aosta-Pre Saint Didier line) always provided by Trenitalia and RFI and referred to advanced wear on the wheel (especially on the wheel tread) and on the rail. In this way other analysis will be carried out in order to further validate the whole model.

Moreover other wear mechanisms in addition to abrasive wear (plastic wear, pitting wear etc.), that may become quite important especially in conditions of advanced wear (in particular on the wheel flange), will be considered within the wear model. Finally, a code optimization, concerning the vehicle model (in particular the global contact model), the wear model and the whole loop, is scheduled for the future in order to reduce the simulation times.

\section{Acknowledgements}

Authors would like to thank Engg. R. Cheli and G. Grande of Trenitalia S.p.A. for providing and giving the permission to edit the data relative both to the vehicle DMU Aln 501 Minuetto and to the wheel wear evolution; a special thanks also goes to the Engg. R. Mele and 
M. Finocchi of Rete Ferroviaria Italiana for the data relative to the Aosta-Pre Saint Didier line.

\section{References}

[1] E. Meli, S. Falomi, M. Malvezzi, A. Rindi, Determination of wheel-rail contact points with semianalytic methods, Multibody System Dynamic 20 (2008) 327-358.

[2] J. Auciello, E. Meli, S. Falomi, M. Malvezzi, Dynamic simulation of railway vehicles: wheel/rail contact analysis, Vehicle System Dynamics 47 (2009) 867-899.

[3] J.J. Kalker, Three-dimensional Elastic Bodies in Rolling Contact, Kluwer Academic Publishers, Dordrecht, Netherlands, 1990.

[4] J.J. Kalker, Survey of wheel-rail rolling contact theory, Vehicle System Dynamics 8 (1979) 317-358.

[5] H. Hertz, The contact of elastic solids, Journal fur Die Reine und Angewandte Mathematik 92 (1881) 156-171.

[6] F. Braghin, R. Lewis, R.S. Dwyer-Joyce, S. Bruni, A mathematical model to predict railway wheel profile evolution due to wear, Wear 261 (2006) 1253-1264.

[7] R. Enblom, M. Berg, Simulation of railway wheel profile development due to wear influence of disc braking and contact environment, Wear 258 (2005) $1055-1063$.

[8] T.G. Pearce, N.D. Sherratt, Prediction of wheel profile wear, Wear 144 (1991) 343-351.
[9] I. Zobory, Prediction of wheel/rail profile wear, Vehicle System Dynamics 28 (1997) 221-259.

[10] T. Jendel, M. Berg, Prediction of wheel profile wear, Supplement Vehicle System Dynamics 37 (2002) 502-513.

[11] P. Toni, Ottimizzazione dei profili delle ruote su binario con posa $1 / 20$, Technical Report, Trenitalia S.p.A., 2010.

[12] S. Iwnicki, The Manchester Benchmarks for Rail Vehicle Simulators, Swets and Zeitlinger, Lisse, Netherland, 1999, 2008.

[13] J.-F. Antoine, C. Visa, C. Sauvey, G. Abba, Approximate analytical mode for hertzian elliptical contact problems, Journal of Tribology 128 (2006) 660-664

[14] K. Johnson, Contact Mechanics, Cambrige University Press, Cambridge, UK, 1985

[15] A.A. Shabana, M.Tobaa, H. Sugiyama, K.E. Zaazaa, On the computer formulations of the wheel/rail contact problem, Nonlinear Dynamics 40 (2005) 169-193.

[16] S. Iwnicki, Simulation of wheel-rail contact forces, Fatigue and Fracture of Engineering Materials and Structures 26 (2003) 887-900.

[17] J.J. Kalker, A fast algorithm for the simplified theory of rolling contact, Vehicle System Dynamics 11 (1982) 1-13.

[18] C. Esveld, Modern Railway Track, Delft University of Technology, Delft, Netherland, 2001, 1985.

[19] Railway applications - in service wheelset operation requirements - in service and off vehicle wheelset maintenance, EN 15313, 2010. 